\title{
MASTER
}

WFPS: TME-79-018

APRIL 1979

UC $20 a$

\section{ANALYSIS OF THE EARLY PHASE OF TOKAMAK \\ PLASMA OHMIC HEATING}

\author{
ERIC VON VALTIER \\ UNIVERSITY OF MICHIGAN
}

COOPERATIVE GRADUATE EDUCATION PROGRAM IN FUSION TECHNOLOGY ADMINISTERED FOR THE U.S. DEPARTMENT OF ENERGY

BY THE WESTINGHOUSE ELECTRIC CORPORATION

CONTRACT EG-77-C-02-4231-AOOO

DISTRIBUTION OF THIS DOCUMENT IS UNLIMITED

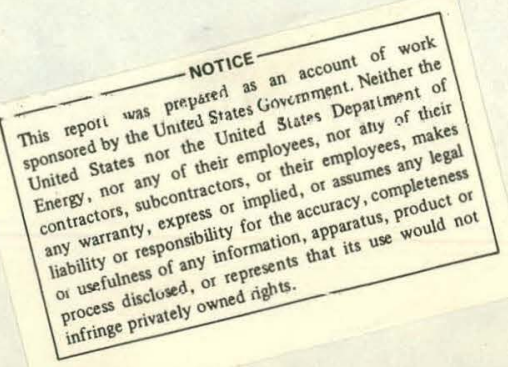




\section{DISCLAIMER}

This report was prepared as an account of work sponsored by an agency of the United States Government. Neither the United States Government nor any agency Thereof, nor any of their employees, makes any warranty, express or implied, or assumes any legal liability or responsibility for the accuracy, completeness, or usefulness of any information, apparatus, product, or process disclosed, or represents that its use would not infringe privately owned rights. Reference herein to any specific commercial product, process, or service by trade name, trademark, manufacturer, or otherwise does not necessarily constitute or imply its endorsement, recommendation, or favoring by the United States Government or any agency thereof. The views and opinions of authors expressed herein do not necessarily state or reflect those of the United States Government or any agency thereof. 


\section{DISCLAIMER}

Portions of this document may be illegible in electronic image products. Images are produced from the best available original document. 
WFPS : TME-79-018

APRIL 1979

ANALYSIS OF THE EARLY PHASE OF TOKAMAK

PLASMA OHMIC HEATING

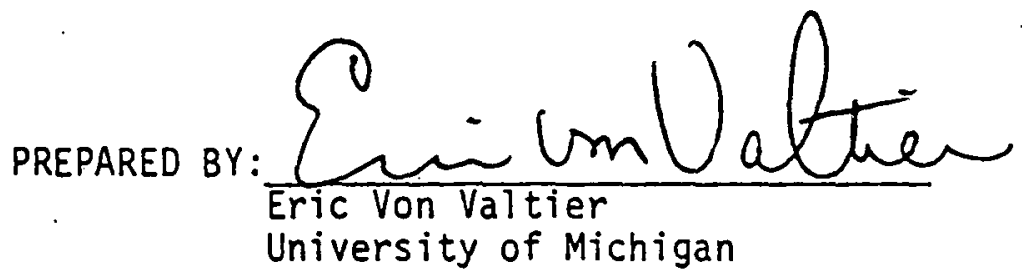

REVIEWED BY: $\frac{\text { Jy, } 3 \text { eck }}{\text { F. M. Heck, Manager }}$

Systems Engineering

Westinghouse Electric Corporation

APPROVED
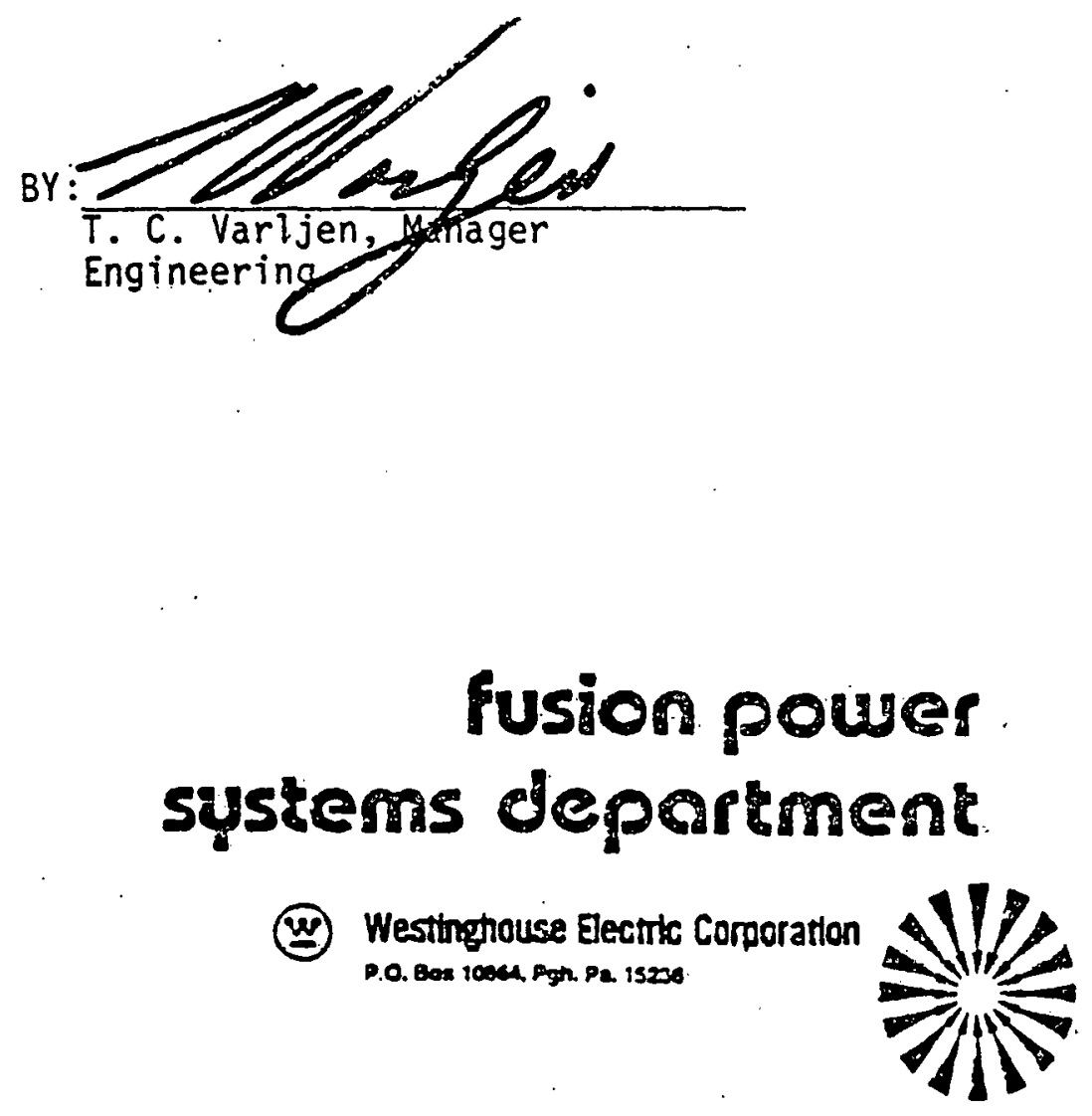


\section{ACKNOWLEDGEMENT}

This work was performed for the U. S. Department of Energy, under Contract EG-77-C-02-4231-A000, Cooperative Graduate Education Program in Fusion Technology. Reproduction, translation, publication, use and disposal, in whole or in part, by or for the United States. Government is permitted.

\section{LEGAL NOTICE}

This report was prepared as an account of Government sponsored work. Neither the United States, nor the Administration, nor any person acting on behalf of the Administration:

A. Makes any warranty or representation, expressed or implied, with respect to the accuracy, completeness, or usefulness of the information contained in this report, or that the use of any infuriliation, apparatus, method or process disclosed in this report may not infringe privately owned rights; or

B. Assumes any liabilities with respect to the use of, or for damages resulting from the use of any information, apparatus, method, or process disclosed in this report.

Printed in the United States of America Available from

National Technical Information Service

U.S. Department of Commerce

5285 Port Royal Road

Springfield, VA 22161

Price: Printed Copy $\$ 5.00$; Microfiche $\$ 3.00$ 


\begin{abstract}
ANALYSIS OF THE EARIY PHASE OF TOKAMAK

PLASMA OHMIC HEATING
\end{abstract}

by

Eric von Valtier

A study of ohmically heated tokamak systems has been performed in which the evolution of the plasma is followed through the initiation period up to 100 ev. A zero-dimensional model of the bulk plasma is utilized, with a one-dimensional model of the plasma inductance. Losses due to ionization and oxygen impurity processes are evaluated using the best available atomic data. The total oH system energy expended in forming and heating the plasma up to a reference temperature of $100 \mathrm{ev}$. is calculated and found to be a function of the maximum induced loop voltage. The latter is found to be limited by thresholds for runaway electron production. Finally, preliminary results are presented on fully one-dimensional calculations which verify rigorously the formation of inverted temperature and current profiles as ealy as $50 \mathrm{eV}$. in the heating process. 
TABLE OF CONTENTS

LIST OF FIGURES . . . . . . . . . . . . . . . . iii CHAPTER

I. INTRODUCTION. . . . . . . . . . . . . 1

1. Motivation

2. Scope

3. Outline of the Analysis

II PHYSICS FORMUATLION • . . . . . . . . 9

1. Basic Physics of Ohmic Heating

2. Formulation of Rate Equations

3. Ionization Rates

4. Atomic Oxygen Processes

5. Electron-Ion Kinetics

6. Electron Runaway

II ELECTRICAL FORMULATION. . . ... . . . 30

1. Magnetic Diffusion

2. Finite-Element Analysis of Magnetic Diffusion

3. $\mathrm{OH}$ Circuitry

IV RESULTS . . . . . . . . . . . . 50

1. Initial conditions

2. Physics Results

3. Electrical Results

4. Diffusion Simulation

APPENDIX A. . . . . . . . . . . . . . . . 84

LIST OF REFERENCES. . . . . . . . . . . . . 88 


\section{INTRODUCTION}

\section{Motivation}

As experiments in magnetically confined CTR research steadily approach the breakeven mark, various groups are beginning to undertake serious design of fusion systems and components. As yet, much of this design work must be performed in an atmosphere of moderate to serious uncertainty about certain key parameters. Since the actual reaction operating conditions have not yet been produced in any laboratory, much of the design work is based upon data obtained by mathematical simulation of the proposed reactor system. Several groups have invested much effort in the implemenation of computational schemes which as accurately as possible model a fusion system. The present study grew out of the author's involvement with several packaged simulation codes, in particular with their application to the analysis of the electrical support systems required in typical proposed tokamak systems.

One significant characteristic of tokamak system simulation became clear after a short time: a diverse range of physical processes, covering many orders of 
magnitude of the corresponding parameters, all interact closely to produce a given physical state of the system. In addition, the dynamic evolution of the system is highly sensitive to certain of these parameters, while being somewhat insensitive to others. Moreover, the relative sensitivities are themselves changing as the reaction enters and leaves various "regimes" of operation.

Despite the prevailing uncertainty about some of the basic fusion physics, several gross features are by now universally accepted in regard to tokamak systems :

A. The physical reactor will be a laboratory grade machine involving extremes of temperature $\left(24^{\circ} \mathrm{K}\right.$. in superconducting magnet coils vs. $210^{10} \circ \mathrm{K}$. in the plasma core), pressure $\left(\sim 10^{-4}\right.$ Torr in the initial gass fill vs. kilonewton stresses in some of the structure), and radiation (intense UV and $\mathrm{X}$-ray as well as $14 \mathrm{Mev}$ neutron fluxes $\left.210^{14}\right)$.

B. The process of energy extraction from a net power producing system will involve technologies which are yet undeveloped. 
C. The system will contain very large circulating electrical powers, possibly in the Gigawatt range.

As the last point above is of particular interest to the author, a systematic study of the electromagnetic aspects of magnetic fusion systems was undertaken. It was found desirable to organize the study into several modules, organized approximately according to the stages of time evolution of a projected operational fusion system. In this report, the results of the first phase of study are compiled, on the basis of which the electrical power requirements for the initiation phase (to be defined in the following section) can be evaluated. The work presented here is an extension of the analyses originially formulated by Duchs and Griem (1), Hawryluk and Schmidt (2), and Breton et al. 732 . Approximately one-half of the present report represents a refinement of the work reported in the previous three references. In the remaining half, original results are presented on startup energetics. based upon the refined calculations.

\section{Scope}

This report covers the first of three parts of a study whose objective is the calculation of the complete set of electrical network variables which 
describe the various electromagnetic processes required to startup and maintain a tokamak discharge. In part I, presented here, a zero-dimensional model of the plasma is simulated in interaction with a rudimentary ohmic heating system, to determine the gross energetics of the plasma formation and subsequent heating. For reference, a tokamak discharge cycle can be conveniently divided (temporally) into four stages:
A. Initiation
B. Ohmic heating/neutral beam injection
C. Maintenance
D. Shutdown

During the initiation phase, which occupies the first several milliseconds of the cycle, cold, neutral gas is pulsed into the evacuated vessel, rapidly ionized, and heated to a temperature of a few ev. The ohmic heating cycle then proceeds, during which the plasma is heated to a temperature of the order of $1 \mathrm{keV}$. by inducing a strong electric field in the plasma volume. At this time, high energy neutral beams of additional fill gas are injected into the plasma, further heating it to reaction temperatures. During the latter part of this phase and all of the following phase, the only function of the ohmic heating system is to maintain a steady plasma current of possibly several megamperes, 
which is required for effective confinement of the plasma in the toroidal magnetic field (which inherently possesses moderately "bad" curvature).

The shutdown phase is presently an unknown area since the best experiments to date have not been able to produce a plasma evolution to this stage. However, it is fairly certain that at the time of shutdown of a fusion cycle, the plasma self-inductance field may contain as much as a gigajoule of magnetic energy which must be dissipated or stored in an orderly fashion. It would be desirable both energetically and physically to discharge this energy back into the oH system energy store.

The present report is concerned solely with phases $A$ and $B$ above, and is concerned with the behavior of the system as it evolves to a temperature of $\sim 100 \mathrm{eV}$. In earlier experiences with tokamak simulation calculations, it was found that during the early part of the discharge a complex set of physical processes control the evolution of th discharge. Moreover, the types of processes involved (i.e., ionization, line radiation, magnetic difusion, etc.) are of a greater influence during the early part of the cycle than during the later stages, which have been studied more extensively for obvious reasons. It was thus decided to separate this phase of operation and subject it to 
a separate study. This separation is of more than pedantic value, due to the mathematical nature of the problem. Specifically, the set of non-linear differential equations required to model a tokamak system is inherently quite stiff. For example, we have had to work with time steps in the range of fractions of a microsecond up to several seconds. In addition to the changing time constants, as the discharge proceeds, the complete appearance and disappearance of certain physical processes, as represented by the corresponding equations, must be tolerated. Hence, it is anticipated that the type of analysis performed in the present, study will appear as a module in a complete system simulation and for this reason it was developed.

As a final introductory note, the following Iimitations of the present study are specified:

A. A zero-dimensional model of the plasma is employed, with a one-dimensional electrical analogue being used for representing its interaction with the $O H$ system.

B. Particle transport is considered in only a rudimentary way.

c. Only the oH system, being the major energy consumer, is considered (i.e., the vertical field system is neglected). 


\section{Outline of the Analysis}

In Chapter II the basic physical processes occuring in the plasma will be analyzed. From this analysis a set of rate equations will be synthesized, whose solution will describe the time evolution of the pertinent plasma parameters. In particular, a great deal of attention will be devoted to the process of impurity poisoning by oxygen. This has been found in previous studies (2) to be significant with respect to proper plasma formation and heating.

These equations will then be solved under simplified electrical assumptions. The results of these calculations will be used as a reference in the later sections.

In Chapter III a more refined electrical model of the plasma will be developed, clearly describing its interaction with the $\mathrm{OH}$ system. Additional calculations will then be performed using this improved model.

Throughout this study, the following set of electrical parameters has been used (5). This set of parameters is believed to be representative of the optimum conditions for a reduced-radius startup of TFTR. 
TABLE 1

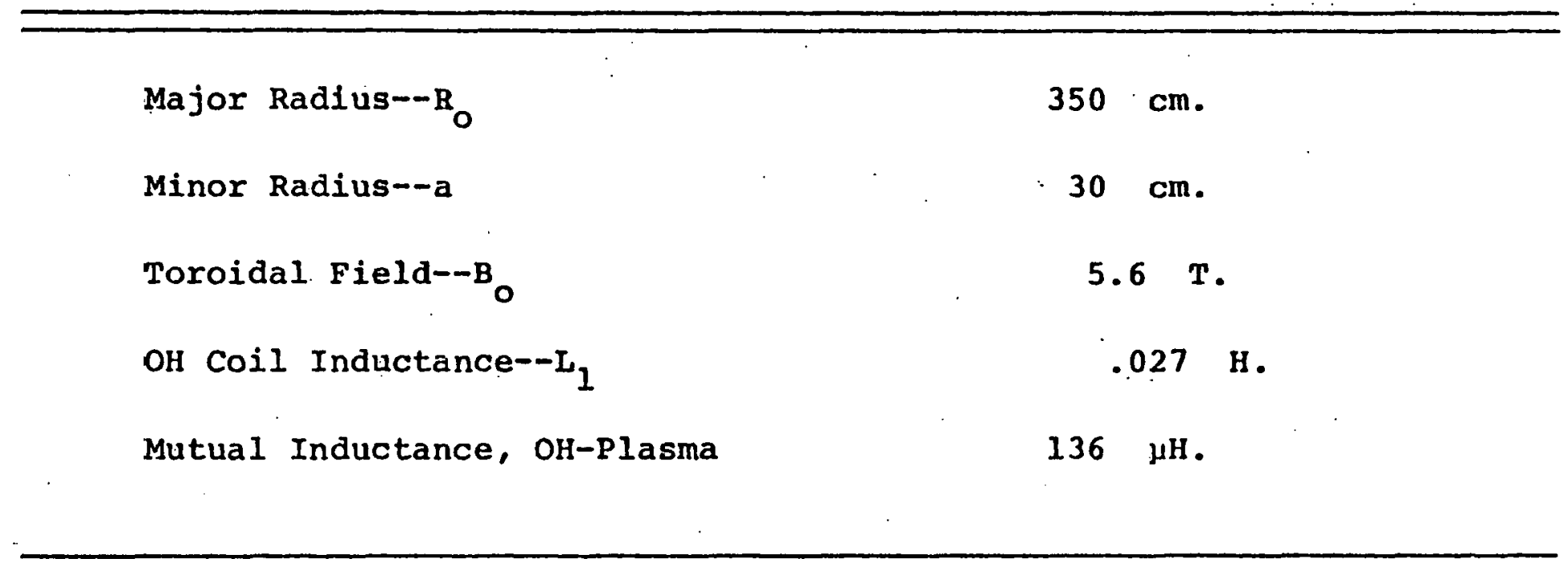




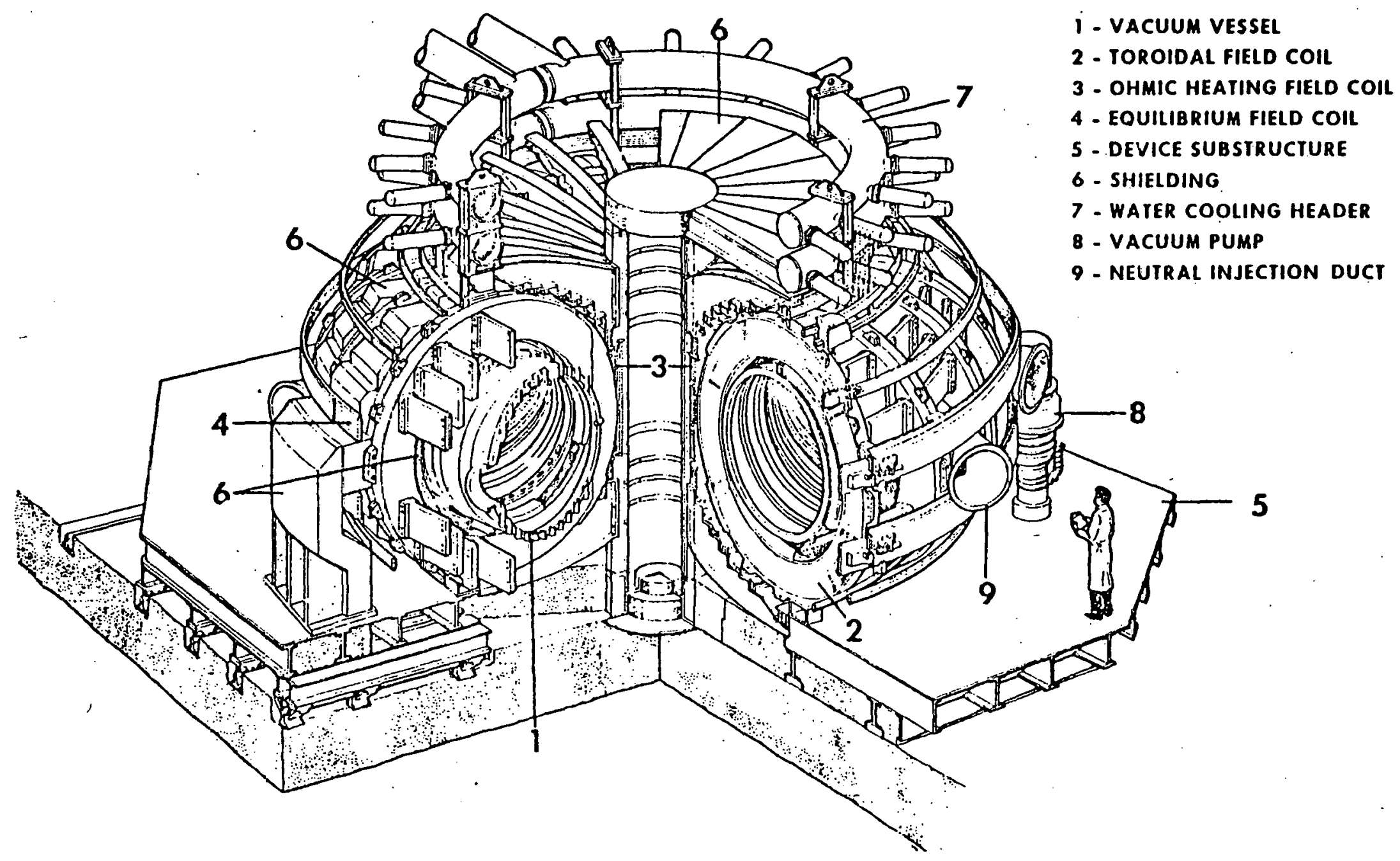

Figure 1. Physical Layout of TFTR 
II. PHYSICS FORMULATION

1. Basic Physics of Ohmic Heating

Figure 2 illustrates the schematic arrangement.

of the $O H$ system as viewed in an axial plane through the plasma:

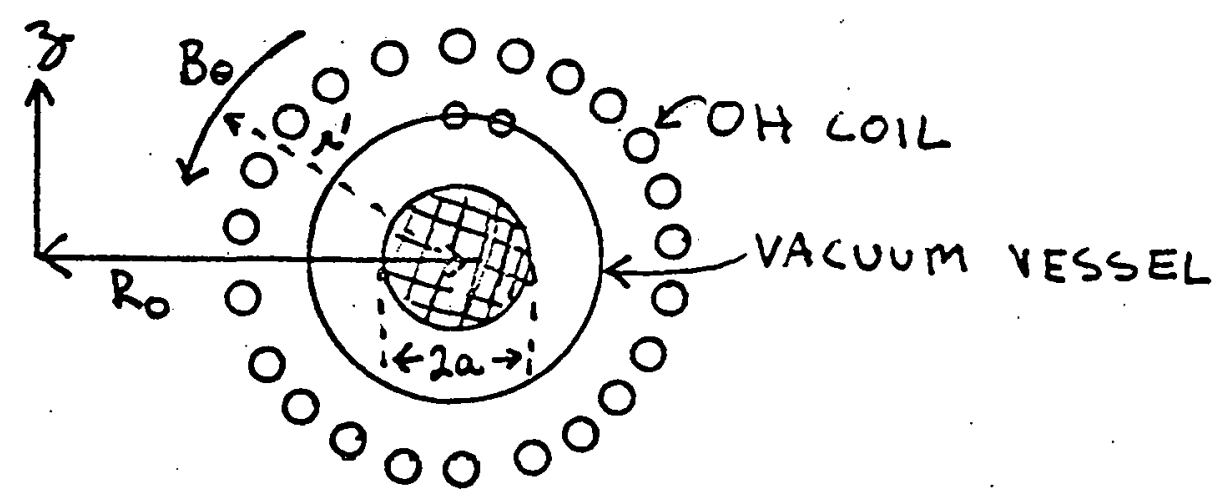

Figure 2. Cross-section view of OH system.

From Ampere's law, the polidal field generated by the $\mathrm{OH}$ coil is calculated as:

$$
\begin{array}{ll}
2 \pi \mu^{\prime} H_{\theta}=I_{0} & I_{O H}=O H \text { COIL } \\
H_{\theta}=\frac{I_{0}}{2 \pi N^{\prime}} &
\end{array}
$$

9 
10

From this, the total oH generated flux

contained within the plasma ring can be calculated to be: $\Phi=\int_{0}^{R_{0}-a} \mu_{0} H_{\theta}(\lambda) \cdot 2 \pi \wedge d \lambda$

$=\int \mu_{0} I_{0} g(\Lambda) 2 \pi \nu d n$

$\Rightarrow \Phi=M I_{0}$

$(2-1)$

where $M$, defined according to equation $(2-1)$, is the mutual inductance between the plasma ring and the $\mathrm{OH}$ coil. Under certain conditions, which will be found to be valid in the present study, this important parameter is a fixed, geometric property of the system. If $I_{0}$ is allowed to vary in time, a toroidal electric field will be induced in the plasma, the value of which is calculated from Faraday's law as follows:

$$
\begin{aligned}
2 \pi R E_{O H} & =-\dot{\Phi}=-m \dot{I}_{0} \\
E_{O H} & =\frac{-m \dot{I}_{0}}{2 \pi R}
\end{aligned}
$$

$(2-2)$

where $R$ is the radius of the toroidal path within the plasma along which it is to be calculated. For a plasma conductivity $\sigma$, the induced current density is: 


$$
\underline{j}=\sigma \underline{E}
$$

and the volumetric heating rate due to this current is:

$$
P=\frac{\dot{\gamma}^{2}}{\sigma}=\left(\frac{m \dot{I}_{0}}{2 \pi R}\right) \sigma
$$

Under a quasi-steady state condition, equation (2-3) would be an exact expression for the ohmic heating rate. However, during transient conditions inductive effects will modify this considerably. These will be considered in some detail further on in the analysis.

\section{Formulation of Rate Equations}

The ohmic heating power derived above represents the only significant source of energy into the plasma electrons (the ions receive essentially no direct ohmic heating power due to their considerably greater mass relative to the electrons), against which must be balanced a number of loss mechanisms. In the present study the following loss processes have been considered:
A. Energy used to ionize $\mathrm{H}^{\circ}$ atoms
B. Energy used to ionize Oxygen atoms of all present ionization states
c. Energy loss due to $\mathrm{H}^{+}$resonant line radiation 
12

D. Energy loss due to $0^{+}$resonant line radiation (all states)

E. Electron-ion coulombic heáting

F. Convective heat loss (ie., particle transport).

Losses due to brehmstrahlung and cyclotron radiation are negligible in the temperature range of interest (1-100 vv.) and are neglected. The resulting equation for electron energy density is:

$$
\begin{aligned}
\frac{3}{2} \frac{d}{d t}\left(m_{e} T_{e}\right) & =I_{p}^{2} R_{p}-Q_{e i}-M_{e} M_{0} R_{1} Q_{H}-M_{e} M_{i} P_{\lambda} \\
& -m_{e} \sum_{i=1}^{g}\left(O_{i} S_{i} Q_{i}+P_{i} O_{i}\right) \\
& -\frac{3}{2} \frac{m_{e} T_{e}}{T_{E e}}
\end{aligned}
$$

$(2-4)$

$$
\begin{aligned}
n_{e} & =\text { density of electrons } \\
n_{i} & =\text { density of } \mathrm{H}^{+} \text {ions } \\
n_{0} & =\text { density of } \mathrm{H}^{\circ} \text { neutrals } \\
R_{1} & =\langle O U\rangle_{i}=\text { ionization rate }\left(\mathrm{H}^{\circ} \rightarrow \mathrm{H}^{+}\right) \\
\varphi_{H} & =\left(\mathrm{H}^{\circ} \rightarrow \mathrm{H}^{+}\right) \text {ionization energy } \\
\mathrm{O}_{i} & =\text { density of oxygen in } i-t h \text { ionization state } \\
s_{i} & =\text { ionization rate for } O_{i} \rightarrow O_{i+1} \\
\varphi_{i} & =\text { ionization energy for }\left(0_{i} \rightarrow O_{i+1}\right) \\
P_{h} & =\text { power loss from line radiation }\left(e \rightarrow H^{\circ}\right) \\
P_{i} & =\text { power loss from line radiation }\left(e \rightarrow o_{i}\right)
\end{aligned}
$$

where all other symbols have their usual meanings. 
13

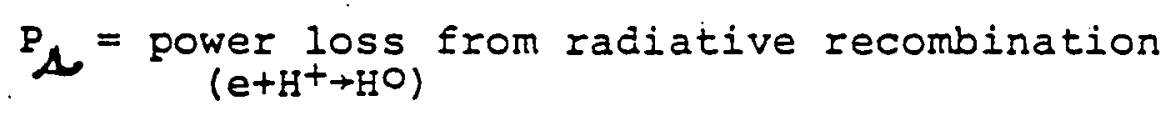

The corresponding energy equation for $\mathrm{H}^{+}$ions is:

$$
\frac{3}{2} \frac{d}{d t}\left(m_{i} T_{i}\right)=Q_{e i}-\frac{3}{2} \frac{m_{i} T_{i}}{T_{E i}}
$$

$(2-5)$

Energy equations for neutrals and Oxygen atoms have not been used as they are of little significance to the problem here; they are assumed to remain cold throughout the calculations.

Equations $(2-4)$ and $(2-5)$ describe the gross energy balance in the plasma, and their solution requires the simultaneous solution of rate equations for all particle densities involved. The equations employed are:

$$
\begin{aligned}
& \frac{d m_{e}}{d t}=m_{e} m_{0} R_{1}-m_{e} m_{i} R_{2}+\sum_{i=1}^{0} S_{i} O_{i}-\frac{m_{e}}{\tau_{p}}(2-6) \\
& \frac{d m_{i}}{d t}=M_{e} M_{0} R_{1}-M_{e} M_{i} R_{2}-\frac{m_{i}}{T_{p}} \\
& \frac{d m_{0}}{d t}=\Gamma-m_{e} M_{0} R_{1}+M_{e} M_{i} R_{2} \\
& \frac{d O_{1}}{d t}=-M_{e} O_{1} S_{1}
\end{aligned}
$$


14

$\frac{d}{d t} O_{2}=m_{e} O_{1} S_{1}-m_{e} O_{2} S_{2}$

$\frac{d}{d t} O_{3}=m_{e} O_{2} S_{2}-m_{e} O_{3} S_{3} \quad(2-11)$

$(2-10)$

$$
(2-11)
$$

$\frac{d}{d t} O_{9}=M_{e} O_{8} S_{8}$

$(2-17)$

The final equation required to close this set is the electrical power balance relation, which must account for the inductive effects due to energy stored in the plasma self-magnetic field. The details of this are derived in Appendix $A$. 


$$
\frac{d I_{p}}{d t}=\frac{V_{0}-I_{p} R_{p}}{L_{\text {int. }}}
$$

where: $I_{p}=$ total plasma current

$R_{p} \quad=$ total plasma resistance

$\mathrm{v}_{\mathrm{o}}=$ applied toroidal voltage

$L_{\text {int }}=$ total internal inductance of plasma Note that we have assumed voltage-source heating of the plasma to maintain consistency with other published work on impurity effects. This restriction will be removed in the final formulation. Further, throughout the study a uniform current profile is assumed. This latter point will be dealt with more completely in section III-1.

The simultaneous solution of equations (2-4) thru (2-18) corresponding to a specified set of initial conditions forms the kernel of the required computations. The equations have been formulated in such a way as to facilitate an explicit form of numerical integration of any desired accuracy. However, the essence of the physical problem lies in the correct evaluation of the various co-efficients appearing in the right-sides of the rate equations. These will next be analyzed in detail. 
As a preface to the following sections, it should be noted that the conditions under which tokamak plasmas are created and subsequently evolve were not readily attainable in the laboratory prior to the current state of intensive CTR research. As a result, much of the required data, as well as the motivation for accompanying theoretical work, is available only from astrophysical observations. Some of this data is believed to be fairly accurate, whereas some is altogether absent. It represents the best available information at this time.

\section{Ionization Rates}

The physics of the ionization process is quite important in a simulation nf plasma formation. In some early simulation work, frequent use has been made of the saha equation for calculating ionization. It is, we feel, quite inappropriate in the present situation due to the presence of strong forces which drive the system away from equilibrium. Griem has argued (12) that the conditions of local-thermodynamic-equilibrium (ITE) should prevail and render the saha equation approximately valid. However, the present results which are based upon explicit kinetic calculations, as opposed to the entirely non-kinetic saha equation, clearly show the latter to be Invalid. 
The ionization states, potentials, and first excited state for the oxygen ions are listed in Table 2 for reference:

TABLE 2

\begin{tabular}{lcclc}
\hline \hline & & \multicolumn{1}{c}{$\begin{array}{c}\text { POTENTIAI } \\
(\mathrm{eV} .)\end{array}$} & \multicolumn{1}{c}{$\begin{array}{c}\text { STATE } \\
\text { ION }\end{array}$} & $\begin{array}{c}\text { TRANSITION } \\
\text { WAVELENGTH }\end{array}$ \\
\hline $0_{I}$ & 0 & 13.6 & $2 \mathrm{~s}^{2} 2 \mathrm{p}^{4} \rightarrow 2 \mathrm{p}^{3} 3 \mathrm{~s}$ & $1303 \mathrm{~A}^{\circ}$ \\
$0_{I I}+1$ & 35.1 & $2 \mathrm{~s}^{2} 2 \mathrm{p}^{3} \rightarrow 2 \mathrm{~s} 2 \mathrm{p}^{4}$ & 833 \\
$0_{I I I}+2$ & 54.9 & $2 \mathrm{~s}^{2} 3 \mathrm{p}^{2} \rightarrow 2 \mathrm{~s} 2 \mathrm{p}^{3}$ & 834 \\
$0_{I V}+3$ & 77.4 & $2 \mathrm{~s}^{2} 2 \mathrm{p} \rightarrow 2 \mathrm{~s} 2 \mathrm{p}^{2}$ & 789 \\
$0_{V}+4$ & 113.8 & $2 \mathrm{~s}^{2} \rightarrow 2 \mathrm{~s} 2 \mathrm{p}$ & 630 \\
$0_{V I}+5$ & 138.1 & $2 \mathrm{~s}^{2} 2 \mathrm{p}$ & 1033 \\
$0_{V I I}+6$ & 739 & $1 \mathrm{~s}^{2}+1 \mathrm{~s} 2 \mathrm{p}$ & $1630 \mathrm{~A}^{\circ}$ \\
$0_{V I I I}+7$ & $>1 \mathrm{keV}$ & not available & \\
\hline
\end{tabular}

The primary data on ionization rates used in the study was obtained from the work of Lotz (7), who utilized a large amount of spectroscopic data and found analytic fits to the ionization cross-sections of the form:

$$
\sigma=\psi_{2} \frac{\ln \left(E / \psi_{1}\right)}{\psi_{1} E}
$$

where the constants $x_{1}$ and $x_{2}$ are given for each element. 
He then averaged the above (energy) differential crosssection over a Maxwellian electron distribution with temperatures in the range of $1-1000 \mathrm{eV}$. The resulting

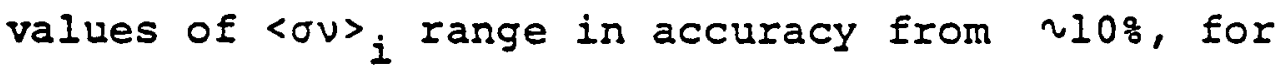
Hydrogen, to $\sim 40$ for the higher oxygen states. Rather than perform additional analytic fits to Lotz's results, we have used them directly in tabular form.

The Lotz results for Hydrogen were compared to the rates for other ionization processes (8), such as neutral-neutral and neutral-ion impact, and the latter were found to be entirely negligible in comparison.

\section{Atomic Oxygen Processes}

Much of the published fusion literature on oxygen impurity effects has utilized the data on line radiation from Duchs and Griem (1) or the more recent work of Duchs, Post, and Rutherford (9). Our research revealed that even the more accurate data of the latter reference was based upon the calculations of Cox and Tucker (10). The C-T calculations, however, are based upon the assumption of coronal equilibrium, in which ionization and recombination produce a detailed-balance type of equilibrium at the specified temperature. It has been our belief since this study began that coronal equilibrium is a poor approximation in transient 
plasmas, such as are found in the initiation phase of a tokamak. The strong electric field rapidly drives the plasma away from equilibrium by allowing ionization to proceed much more rapidly than recombination. Therefore, in our calculations the on-line calculation of line radiation losses, recombination rates, and recombination radiation rates have been performed using the formulations of Cox and Tucker. The energy loss rate due to radiative decay of the collisionally excited state $n^{\prime}$ back to the ground state $n$ ( $n^{\prime}$ 's denote principal quantum numbers) is given by: $\frac{d^{2} E}{d t d V} z^{n, z}=:$

$2.52 \times 10^{-17} m_{z, z} \sum_{m^{\prime}} f_{m n^{\prime}} g_{m m^{\prime}} \frac{e}{T^{1 / 2}}$

$$
\begin{aligned}
& \left(\operatorname{lng} \cdot \mathrm{cm}^{-3} \cdot \operatorname{sen}^{-1}\right) \\
& T \text { in } 0 K
\end{aligned}
$$

in which $\mathrm{n}_{\mathrm{z}, \mathrm{z}}$ denotes the density of atoms of nuclear charge. $z$ and electronic charge $z, f_{n n}$ is the dipole oscillator strength for the transition, and $g_{n n}$ is the free-bound Gaunt factor. For the latter we have utilized the values proposed by Tucker and Gould (13) and listed here for reference: 


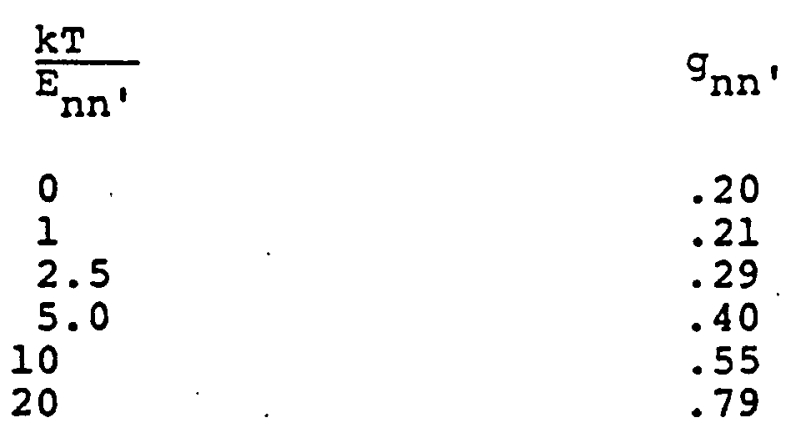

Equation (4-1) : was sumed over all listed transitions in the NBS Table of Atomic Transition Probabilities (14) for $O_{I}-O_{V I I I}$ and $H^{\circ}$. No data was available for $O_{I X}$ and in view of the fact that the ionization energy for $0_{V I I I} \rightarrow 0_{I X}$ is $\sim 1 k e v$, the presence of $0_{\text {IX }}$ was neglected in the calculations. (This data could become important, however, in later work in the kev. range.)

The calculation of the loss rate due to neutral Hydrogen excitation has been somewhat problematic and is a residual source of uncertainty in the present results. Due to the much higher density of Hydrogen as compared to oxygen contamination, it is doubtful that the plasma is transparent to emitted Hydrogen radiation, and probably considerable reabsorption occurs. To properly account for this effect, the associated radiation transport problem must be solved as outlined by Holstein (15), necessitating the inclusion of an additional integral equation involving an escape probability integral of the type: 
21

$$
I(\nu)=\int k\left(\nu, \nu_{0}\right) d \nu_{0}
$$

$(4-2)$

The result of this has a two-fold significance:

(1) the inherently spatially dependent nature of this problem is further amplified, and (2) the effect of radiation imprisonment can seriously perturb the ground state population, requiring one or more additional rate equations to solve. This latter point would imply a need for some difficult to obtain data on excited states of hydrogen, which could probably be calculated with some effort.

We have left this question open and used the following approach in order to be able to complete some calculations. It is assumed that most of the Hydrogen line radiation is reabsorbed, allowing only a small amount of surface escape, without accounting for perturbation of the ground state. This is accomphished through the artifact of attaching an "effective loss co-efficient" to the term in equation (2-4) which accounts for this effect:

$$
P_{\substack{\text { Loss } \\\left(H 0^{\circ}\right)}}=-A_{8} P_{H} m_{e} m_{0}
$$


22

with a typical value for $A_{8}$ of .05 . The magnitude of this problem is in Figure 3, where the value of ne $e_{0} P_{h}$ (ie. the "true" loss rate) is shown from a typical simulation: This data shows that the loss rate exceeds the total power input by nearly an order of magnitude over most of the ionization-buildup range! If a significant fraction ( $25 \%$ ) of this energy escaped, the plasma ionization and heating would be seriously affected.

Hawryluk and Schmidt (16) have suggested that this effect can be properly handled by adding a . correction of a few $\mathrm{eV}$. to the $\mathrm{H}^{\circ}$ ionization energy, but no physical explanation was offered. We have found that this type of correction only slightly modifies the results. Hopefully this problem will be solved in our further investigations.

For the recombination rates, the approximate result of cox and Tucker has been utilized:

$$
\begin{gathered}
R_{z}=2.07 \times 10^{11} z^{2} T^{-1 / 2}(0 \mathrm{~K})\left(.43+\ln \beta^{1 / 2}+.47 \beta^{-1 / 3}\right) \frac{\mathrm{cm}^{6}}{\mathrm{sec}} \\
\beta=z^{2} \frac{\varphi_{H}}{\mathrm{kT}}
\end{gathered}
$$

$(4-3)$ 

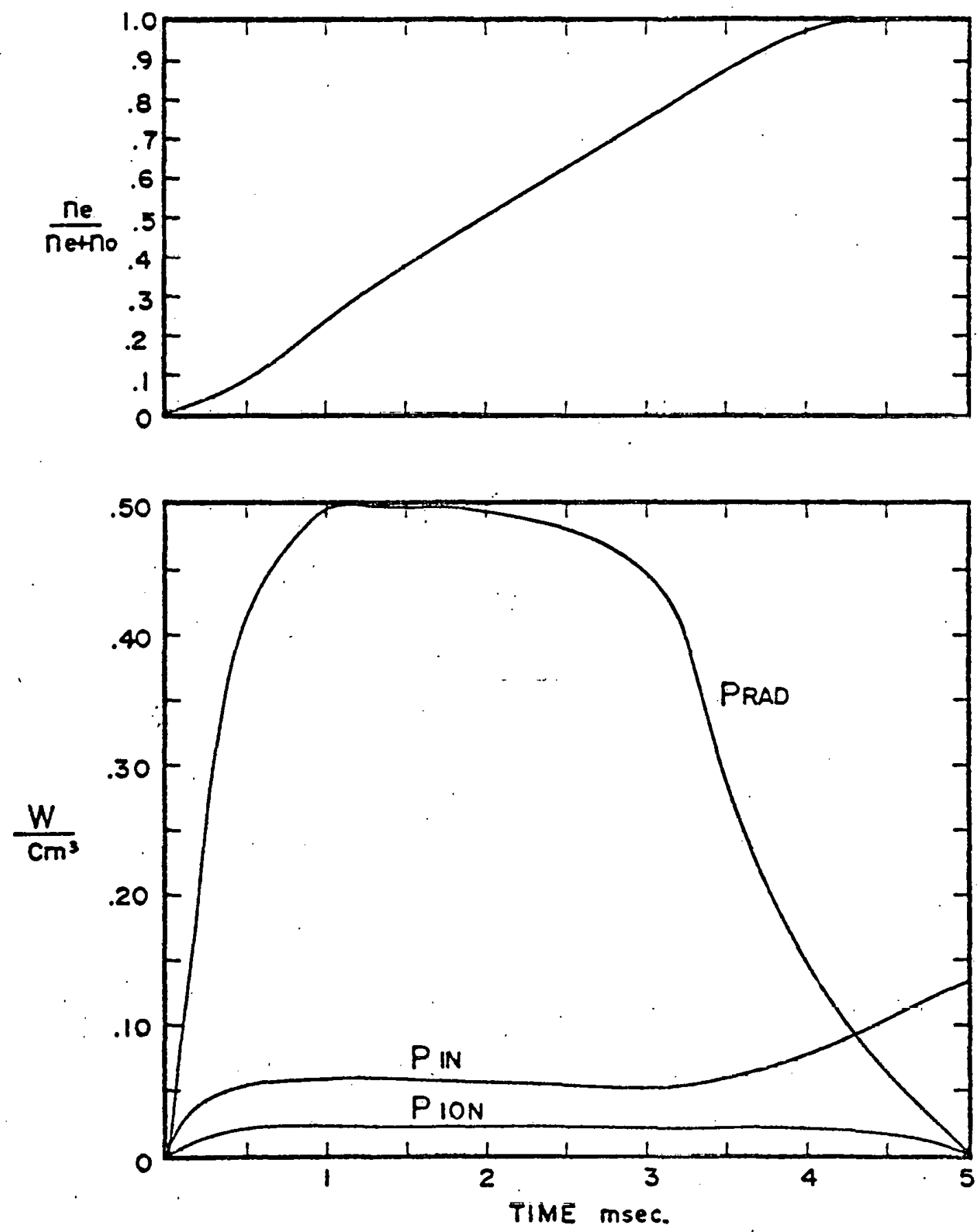

Figure 3. Ionization fraction and power flows vs. time for discharge of Figure 15 (100 vo case). $\mathrm{P}_{\text {in }}=$ resistive power into plasma, $\mathrm{P}_{\text {ion }}=$ poher to ionize $\mathrm{H}^{\circ}, \mathrm{P}_{\text {rad }}=$ power loss from $H^{\circ}$ line radiation calculated from (equation $4-1$ ). 
As pointed out by those authors, this result is strictly valid only for Hydrogenic ions. They present a very complex correction for non-Hydrogenic atoms, which hand calculations reveal to be a very mild corfection. In view of this and the following discussion, equation (4-3) was utilized in the form shown.

A fair amount of work on the process of dielectronic recombination has appeared in the astrophysical literature recently (11). Our opinion is that the published results are fairly approximate in nature, with several levels of approximation in the 10-50\% range appearing in each result. Moreover, the calculated rates (which are rather difficult to compute) tend to be of the same order of magnitude as the more well-established radiative recombination rate of equation $(4-3)$. Also, we have found the results of our calculations to be somewhat insensitive to recombination because, as noted before, the strong ohmic heating field tends to drive the electron temperature so rapidly that ionization dominates recombination by a gond margin.

The loss rate due to radiative recombination was estimated by means of the somewhat involved result of cox and Tucker: 
25

$$
\begin{aligned}
& \frac{d^{2} E_{z, z}^{m}}{d t d V}=: \\
& M_{e} M_{z, z} I_{z_{1,3-1}}^{m}\left[R_{z}+2 A v X\left(I_{z_{1,-1}} / h T\right)\right]
\end{aligned}
$$

where: $I_{z, 3-1}^{m}=$ ioniegtim energy of stats $m$

$$
v=\left(\frac{2 k T}{\pi m_{e}}\right)^{1 / 2}
$$

where the function $X(T)$ is related to the spitzes $\varnothing$-function (17). We have found this loss rate to be negligible in comparison to the other losses and it was not utilized in the final calculations.

5. Electron-Ion Kinetics

For the rate of electron-ion heating, the classical expression has been used for $Q_{e-i}(6)$ :

$$
Q_{e i}=1.2 \times 10^{-28} \frac{T_{i}}{T_{e}^{2 / 2}}\left(\frac{T_{e}}{T_{i}}-1\right) M_{e} M_{i} \ln \Lambda\left(\frac{w}{\mathrm{~cm}^{3}}\right)
$$

when:

$\left(T \sin e V_{1}\right)$

$$
\Lambda=\frac{1.83 \times 10^{16} T_{e}^{3 / 2}}{M_{e}^{1 / 2} Z_{*}}
$$

In the resistivity calculations, classical resistivity has been assumed as well as a uniform current profile:

$$
M_{s}=5.2 \times 10^{-3} Z_{\text {eff }} T^{-3 / 2} \ln \Lambda \quad(\Omega \cdot(\mathrm{cm})
$$


26

At low values of ionization, the electron density and temperature are so low that electron drift is controlled by electron-neutral scattering. The resistivity is thereby enhanced by a correction factor for neutral scattering of the form:

$$
\eta_{a}=\frac{m_{e} \nu_{e \cdot m}}{M_{e} e^{2}}
$$

uteri: $\nu_{e-m}=M_{0} v_{\text {Th. }} \sigma_{e-m}$

For the elastic electron-neutral cross-section the expression used by Ducks and Grieg was employed:

$$
\sigma_{e-m}=10^{-16}\left[\frac{47.4}{E(e v)+1.3}+2.14\right] \mathrm{cm}^{2}
$$

$(5-3)$

finally obtaining:

$$
M_{a}=.21 \frac{M_{0}}{m_{e}} T_{e}^{1 / 2}\left[\frac{47.4}{T_{e}+1.3}+2.14\right](\Omega \cdot \mathrm{cm})
$$

The sum of equations (5-2) and (5-4) is'used for the total value of the resistivity vs. temperature.

6. Electron Runaway

Since its first observation in early stellarator discharges (18), electron runaway has been a serious 
concern in plasma initiation studies. Simply stated, electron runaway occurs when the electron collision frequency is so low that the applied electric field can accelerate an electron to very high energies in less than one collision time. This is likely to occur under conditions of low density and high electric field, precisely the conditons present during the initiation phase of a tokamak discharge. In fact, this probably poses the most severe limitation upon a plasma initiation cycle.

In the original work on runaway, Dreicer (19) proposed the concept of a critical field, $E_{C r^{\prime}}$ above which solutions of the electron Fokker-Plank equation ceased to be stationary. However, as later clarified by Dreicer himself $(20)$ and further refined by Gurevich (21), this limit (which is commonly referred to in the fusion community as the "Dreicer Field") is somewhat over-opt1mistlc; runaway can actially begin to become a significant factor for values of $E / E_{C r} 1$. Moreover, during initiation there is a substantial amount of electron-neutral scattering, a factor which was not considered in the original Dreicer paper, as he assumed that the plasma was fully ionized.

In the more refined analysis of Gurevich, both elastic and inelastic scattering by neutrals were 
28

considered in the collision operator used in the solution of the electron equation. The resulting criterion for the critical field was modified to read as follows:

$$
E_{a_{1}}=2.6 \times 10^{-13} \frac{\mathrm{Me}}{T_{e}} \ln \left[\frac{4 \times 10^{9} T_{e}^{1 / 2} m v_{a}^{2}}{M_{a}^{1 / 2}}\right]_{T_{e} \text { m i neV. }}\left(\frac{v}{\mathrm{~cm}}\right)
$$

$(6-1)$

in the limit of full ionization. For the neutral dominated opposite limit, the result is:

$$
E_{a_{1}}=7 \times 10^{-15} m_{0}\left(\frac{v}{c m}\right)
$$

$(6-2)$

More recent studies have analyzed the effects of runaway electrons in greater detail, to the extent that a rather vast literature on the subject has already developed. A complete listing of the available litertore is contained in the work of Moke (33). It was assumed at the outset of this study that the presence of runaways was of minimal significance provided applied electrical fields were held below the limits given by equations $(6-1)$ and $(6-2)$. However, as the study progressed and this issue was studied more carefully, its significance was seen to be of great concern. In particlar, the results of. sponge (22) show that at relatively low 
values of electric field, the plasma can evolve to a configuration in which essentially all of the plasma current is carried by a filamentary, relativistic electron ring. Whether such a configuration can further evolve into a useful reactor plasma configuration is not clear at present.

Thus, the present results must be viewed as dependent upon the assumption that a plasma can be formed. without the associated formation of a significant relativistic electron current channel. The validity of this assumption will be the subject of further investigation by the author. 
III. ELECTRICAI FORMULATION

\section{Magnetic Diffusion}

The process of magnetic diffusion in plasmas has received only rudimentary attention in most published work to date. It has been recognized for some time that this phenomenon can have important consequences in plasma operation, such as the formation of skin currents and inverted temperature and/or density profiles. The latter are serious in that they can drive certain resistive-tearing modes of instability (23). The physical origin of this process can be readily deduced from Maxwell's equations as follows:

$$
\text { m4: } \begin{aligned}
\underline{\nabla} \times \underline{H}=\underline{\dot{j}} \\
\text { yf } \dot{j}=\sigma \underline{E}, \\
E=\frac{\dot{j}}{\sigma}=\frac{1}{\sigma} \underline{\nabla} \times \underline{H}=\frac{1}{\mu \sigma} \nabla \times \underline{B}
\end{aligned}
$$


31

$$
\begin{aligned}
& \text { mi: } \underline{\nabla} \times \underline{E}=\underline{\nabla} \times\left(\frac{1}{\mu \sigma} \underline{\nabla} \times \underline{B}\right)=-\frac{\partial \underline{B}}{\partial t} \\
& \frac{1}{\mu \sigma} \underline{\nabla} \times(\underline{\nabla} \times \underline{B})=-\frac{\partial \underline{B}}{\partial t} \\
& \frac{1}{\mu \sigma} \nabla(\underline{\nabla} B)-\frac{1}{\mu \sigma} \nabla^{2} \underline{B}=-\frac{\partial B}{\partial t}
\end{aligned}
$$

Since $\underline{\nabla} \cdot \underline{B}=0$ :

$$
\frac{1}{\mu \sigma} \nabla^{2} \underline{B}=+\frac{\partial \underline{B}}{\partial t}
$$

$(7-2)$

where we have neglected all spatial and time variation of $\mu$ and $\sigma$. Equation $(7-2)$ can be recognized as a classical diffusion equation, which in this case describes the diffusion of a magnetic field into a medium of conductivity $\sigma$ and permittivity $\mu$. The normal diffusion constant $\mathrm{k}^{2}$ is given in this case by:

$$
\mathrm{x}^{2}=\frac{1}{10}
$$

Consider the application of equation (7-2) to a cylindrical symmetric conductor, which is approximately true for a toroidal plasma in the large aspect ratio limit. If a toroidal electric field is applied as a step function at $t=0$, the evolution of the magnetic field within the plasma will proceed as depicted in. Figure 4. 


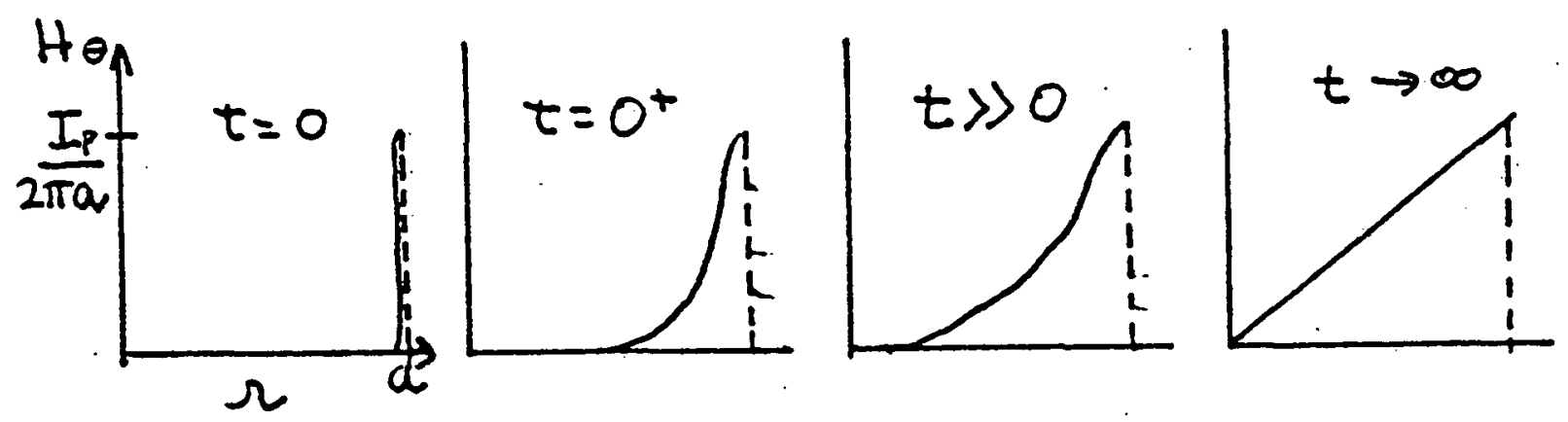

Figure 4. $B_{\ominus}(I)$ at various stages of diffusion following application of a step voltage at $t=0$

The approximate time scale for this sequence can be estimated from the well established results of diffusion theory (24), in which it is shown that an initial $\delta-$ function distribution of source in a diffusing medium of diffusion constant $\mathrm{k}^{2}$ relaxes to a Gaussian spatial distribution of width $I$ in a time period given by:

$$
\tau=\frac{L^{2}}{4 k^{2}}
$$

which for the present magnetic case is:

$$
\tau=\frac{\mu \sigma L^{2}}{4}
$$


From equation (5-2) we can estimate:

$$
\sigma=\frac{1}{\eta_{s}} \simeq 20 T^{+3 / 2}
$$

Substituting this in $(7-3)$ yields:

$$
\tau \simeq 4 \pi \times 10^{-9} \times 20 T^{3 / 2} \frac{L^{2}}{4} \simeq 10^{-7} T^{3 / 2} L^{2}
$$

Considering a plasma of $30 \mathrm{~cm}$. minor radius, at $\mathrm{T}_{e}=100 \mathrm{eV}$.

$$
\tau=.1 \mathrm{sec} .
$$

and at $\mathrm{T}_{\mathrm{e}}$ of $10 \mathrm{eV}$.

$$
\tau \simeq .003 \mathrm{sec} .
$$

These numbers represent the rate at which the magnetic field, and therefore the enclosed current which is related to it by Ampere's law, would diffuse into the plasmas. The implications are fairly clear: at temperatures in the range of $1-100 \mathrm{eV}$. plasma heating is likely to be affected by the finite time required for the applied field to actually reach the interior parts of the plasma. As the temperature increases beyond $100 \mathrm{eV}$. , equation (7-4) shows that the problem becomes increasingly serious, with the outer layers of plasma effectively heating up more rapidly and partially shielding the inner regions from the $\mathrm{OH}$ field. The 
OH system is, at best, only moderately efficient in transferring energy to the plasma in the first place. The preceeding simple estimates are intended only to illustrate the basic nature of transient inductive processes in distributed electrical conductors such as plasmas. The only rigorous method for dealing effectively with these self-inductive field effects is to solve equation $(7-2)$ as a boundary value problem, where an appropriate boundary would be the surface of the plasma at which the relation

$$
H_{\theta}(a)=\frac{I_{p}}{2 \pi a}
$$

would hold. Investigations of early plasma behavior using this approach have recently been undertaken at PPPL (25). However, no formal results have yet been published. Since it was felt that this aspect of the plasma electromagnetics is potentially of some importance, the formulation presented in the following sections was developed. In our approach, rather than trying to simulate equation (7-2), we proceed from the basic electromagnetic description of induction in the plasma in a finite-element form from the beginning. 


\section{Finite-Element Analysis of Magnetic Diffusion}

The formulation starts from the basic induction equations for electric and magnetic fields, and leads naturally to a description of the plasma as a lumpedparameter electrical network. This approach is naturally suited to implementation of computer coded analysis, which is essential due to the time-varying nature of the resultant circuit parameters, particularly $\sigma \mathrm{r}^{3 / 2}$.

We consider a plasma possessing cylindrical symmetry along the toroidal axis of the co-ordinate system used, as shown in Figure 5:

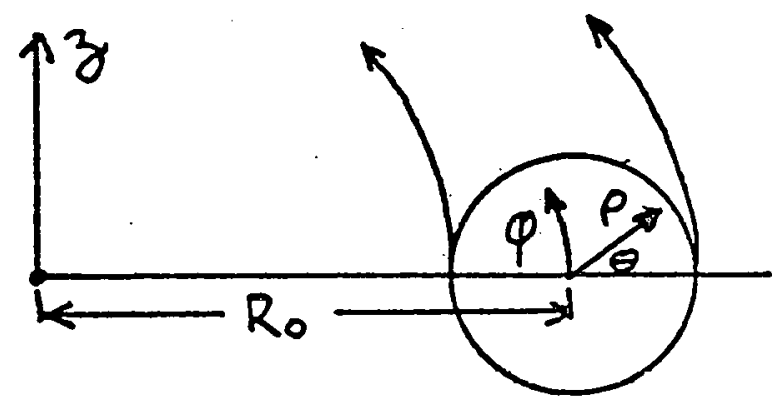

Figure 5. Toroidal co-ordinate system.

It has been shown (28) that the cylindrical approximation leads to errors of order $\varepsilon^{2}$ in inductance and resistance calculations, where $\varepsilon$ is the inverse aspect ratio. The cross section of the plasma is then divided into $n$ concentric shells, each of thickness $h_{j}$ and radius $a^{\prime}$ and characterized by a conductivity $\sigma_{j}$ and permittivity $\mu_{j}$. The magnetic induction in the $j$-th shell (we refer to the magnetic induction of the poloidal field generated 
36

by the plasma itself) is calculated by means of the line integral along the path shown in Figure $6 a$.

$$
\begin{aligned}
& 2 \pi a_{j} H_{j}=\sum_{i=1}^{j-1} I_{j} \\
I_{j} & =\sigma_{j} E_{j}\left(2 \pi a_{j} h_{j}\right) \\
\Rightarrow \quad H_{j} & =\frac{1}{a_{j}} \sum_{i=1}^{j-1}\left(\sigma_{i} a_{i} h_{i}\right) E_{i} \quad(8-1)
\end{aligned}
$$

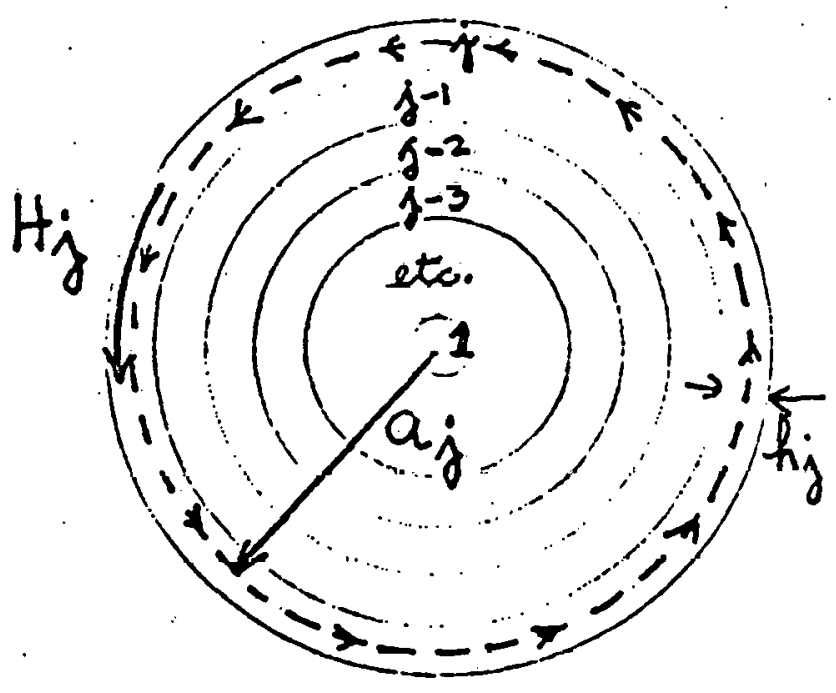

Figure ba. Cross-sectional view of plasma showing division into concentric shells. 
37

Next, the electric field within each shell is calculated by means of a line integral in the equatorial plane shown in Figure $6 b$.

$$
\begin{aligned}
& 2 \pi R^{\prime}\left(E_{j-1}-E_{j}\right)=-\frac{\partial}{\partial t} B_{j} S_{j}=-\mu_{j} \dot{H}_{j}\left(2 \pi R^{\prime} h_{j}\right) \\
& V_{j}-V_{j-1}=\mu_{j} \dot{H}_{j} h_{j}\left(2 \pi R^{\prime}\right) \quad(8-2)
\end{aligned}
$$

when $V_{j}=2 \pi R^{\prime} E_{j}$

$=j$-el toroidal loop voltage

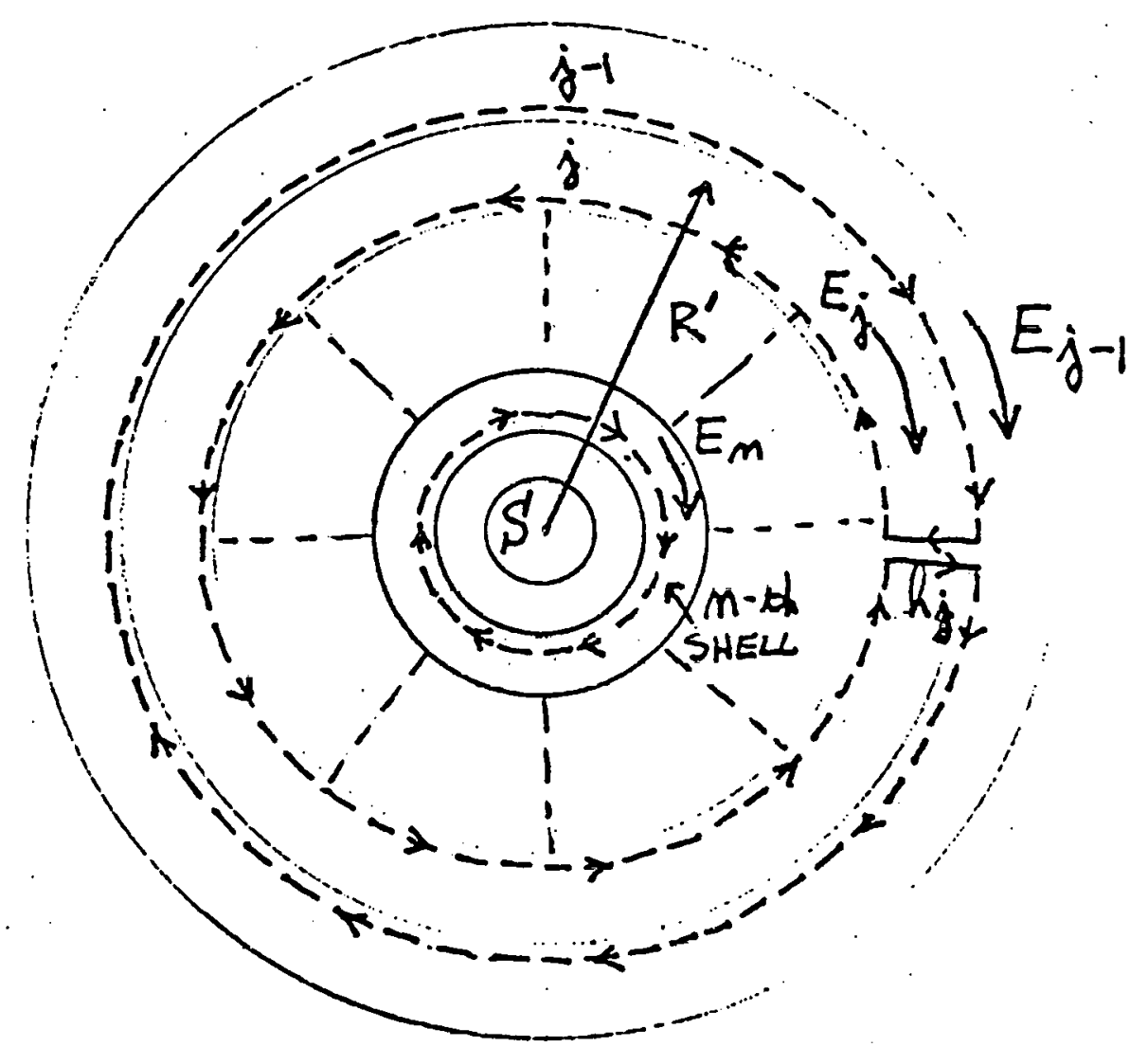

Figure bb. View of equatorial plane showing integration paths for shell voltages. 
38

If we now substitute from equation (8-1) for $\mathrm{H}_{j}$, the following results

$$
V_{j}-V_{j-1}=\frac{\mu_{j} h_{j}}{a_{j}} \sum_{i=1}^{j-1} \sigma_{i} a_{i} h_{i} \dot{V}_{i}
$$

Noting that the total electrical resistance of the $j-t h$ shell is (to within error $\varepsilon^{2}$ )

$$
R_{j}=\frac{1}{\sigma_{j}} \cdot \frac{2 \pi R_{0}}{2 \pi a_{j} h_{j}}=\frac{R_{0}(\text { maj }}{\sigma_{j} a_{j} h_{j}}
$$

then $(8-3)$ can be written as

$$
V_{j}-V_{j-1}=\frac{\mu_{j} h_{j}}{a_{j}} R_{j}^{j-1} \sum_{i=1}^{\dot{V}_{i}} \frac{\dot{R}_{i}}{R_{i}}
$$

or in terms of the toroidal loop voltage in the $f$-th shell:

$$
\left(V_{j}-V_{j-1}\right) \frac{a_{j}}{\mu_{j} h_{j} R_{0}}=\sum_{i=1}^{j-1} \frac{\dot{V}_{i}}{R_{i}}
$$

$(8-4)$

Before proceeding, it should be noted that this analysis is based upon the basic design of TFTR, in which the OH coil system is designed to minimize leakage flux. That is, the closed of flux lines completely encircle the vacuum vessel, with essentially no flux passing directly through the plasma. This can be observed in the flux map of the OH coil in the TRTR Design Study (27). 
39

For other coil geometries, the situation will be somewhat complicated by leakage. Finally, the inner loop integral in Figure bb. is evaluated as:

$$
\begin{aligned}
2 \pi R^{\prime} E_{M} & =-\dot{\Phi}_{P}-\dot{\Phi}_{O H} \\
& =-\frac{\partial}{\partial t} \int\left(\underline{B}_{P}+\underline{B}_{O H}\right) \cdot \underline{d S}
\end{aligned}
$$

where $S$ denotes the surface bounded by the loop. The flux passing through this surface contains two contributions:

$$
\Phi_{p}=L_{\text {ext }} I_{p}=L_{\text {eft }} \sum_{i=1}^{n} \frac{V_{i}}{R_{i}}
$$

$(8-5)$

which is the flux due to the plasma current itself, as characterized by $I_{\text {ext }}$ as shown in Appendix $A$. The other contribution is the flux through $s$ due to the OH current, which is simply

$$
\Phi_{O H}=m I_{0}
$$

$(8-6)$

by definition of M. Combining these we obtain the relation for the surface voltage:

$$
V_{m}=-L_{\text {ext }} \sum_{i=1}^{m} \frac{\dot{V}_{i}}{R_{i}}-M \dot{I}_{0}
$$

Equations $(8-4)$ and $(8-7)$ now constitute a set of $n+1$ simultaneous differential equations for the $n$ 
voltages (currents) in the $n$ plasma shells and the $\mathrm{OH}$ coil. Later we will require an expression for the $\mathrm{OH}$ terminal voltage which is simply derived by summing the EMF's around the coil:

$$
\begin{aligned}
& V_{O H}+L_{O H} \dot{I}_{0}+M \dot{I}_{P}=0 \\
\Rightarrow & V_{O H}=-L_{O H} \dot{I}_{0}-m \sum_{i=1}^{m} \frac{\dot{V}_{i}}{R_{i}}
\end{aligned}
$$

We can now simply "drive" the plasma with a voltage applied to the $\mathrm{OH}$ coil terminals.

To facilitate the analysis of the system under various types of excitation applied to the $\mathrm{OH}$ coil, the electrical network of Figure 7 was synthesized. To establish formally the analogy between this network and the plasma/OH system, the network differential equations are first derived in a straight forward fashion:

$$
\begin{aligned}
V_{j}-V_{j-1} & =L_{j} \dot{I}_{L_{j}} \\
\text { where: } I_{L j} & =\sum_{i=1}^{j-1} \frac{V_{i}}{R_{i}} \\
\Rightarrow \quad \frac{V_{j}-V_{j-1}}{L j} & =\sum_{i=1}^{j-1} \frac{\dot{V}_{i}}{R_{i}} \quad(8-9)
\end{aligned}
$$




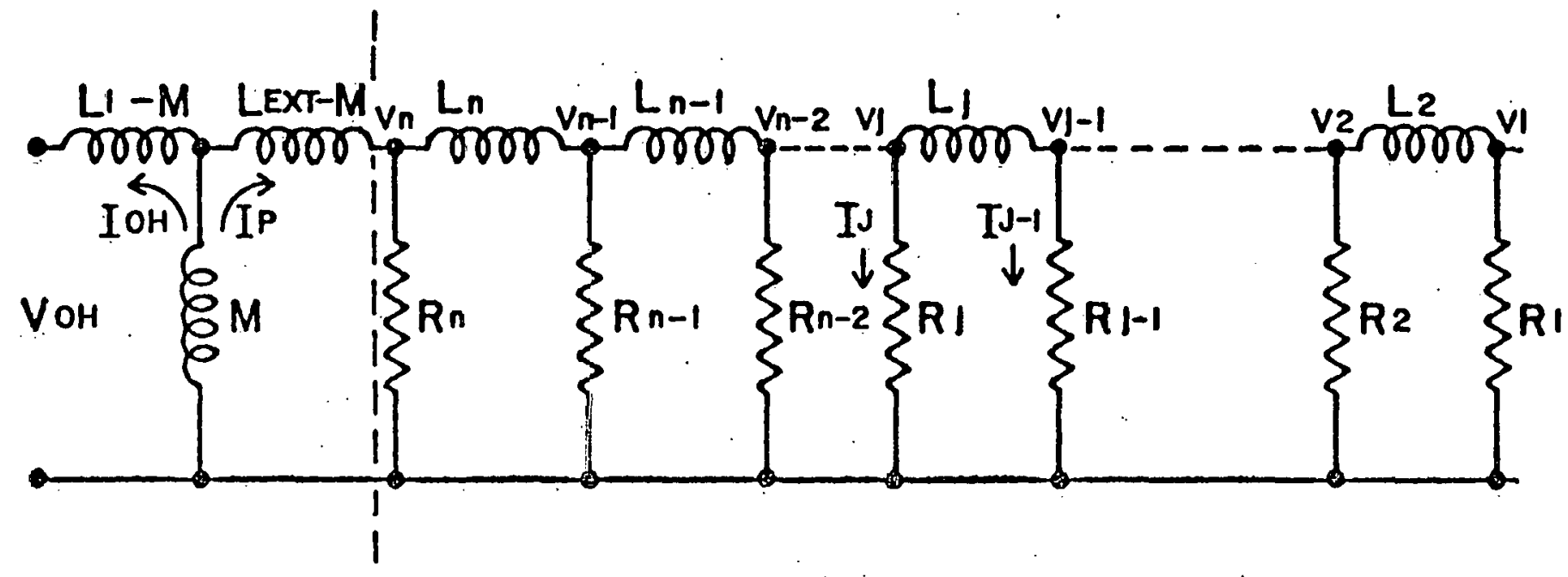
the coupled system of plasma and ohmic heating coil. 
The two input meshes are described by

$$
V_{m}=-m \dot{I}_{0}-L_{\text {eft }} \sum_{i=1}^{m} \frac{\dot{V}_{i}}{R_{i}}
$$

and:

$$
V_{O H}=-L_{1} \dot{I}_{0}-m \sum_{i=1}^{m} \frac{\dot{V}_{i}}{R_{i}}
$$

The analogy is established by noting the equality of equation (8-9) with (8-4) and making the identifications:

$$
\begin{aligned}
& L_{j}=\frac{\mu_{j} h_{j} R_{0}(\text { maj. })}{a_{j}} \\
& R_{j}=\frac{R_{0}(\text { maj. }}{\sigma_{j} a_{j} h_{j}}
\end{aligned}
$$

as well as the equality of equations $(8-10)$ and $(8-11)$ with $(8-7)$ and $(8-8)$ respectively.

As a final step the network equations are manipulated into explicit time-difference form, to facilitate their coding in the numerical integration method used, and a transformation to current variables is made: 
43

$$
\begin{aligned}
& \dot{I}_{1}=\frac{R_{2}}{L_{2}}\left[I_{2}-\frac{R_{1}}{R_{2}} I_{1}\right] \\
& \dot{I}_{2}=\frac{R_{3}}{L_{3}}\left[I_{3}-\frac{R_{2}}{R_{3}} I_{2}\right]-\dot{I}_{1}
\end{aligned}
$$

$$
\begin{aligned}
& \dot{I}_{j}=\frac{R_{j+1}}{L_{j+1}}\left[I_{j+1}-\frac{R_{j}}{R_{j+1}} I_{j}\right]-\dot{I}_{j-1} \\
& \vdots \\
& \dot{I}_{m}=\frac{R_{m-1}}{L_{m}} I_{m-1}-\frac{m}{L_{\text {opt }}} \dot{I}_{0}-\frac{R_{m}}{L_{m}}\left(1+\frac{L_{m}}{L_{\text {ext }}}\right) \\
& \dot{I}_{0}=\frac{1}{\left(m^{2}-L_{1} L_{\text {Exr }}\right)}\left[L_{\text {est }} V_{\text {OH }}+m V_{m}\right]
\end{aligned}
$$

$(8-13)$ 


\section{OH Circuitry}

In this section the circuitry which drives the OH coil will be considered in further detail. The function of this curcuitry is to control the OH coil voltage and current, which in turn controls the plasma current and subsequent plasma evolution. The general form of the $\mathrm{OH}$ current waveform throughout a cycle is as shown in Figure 8 .

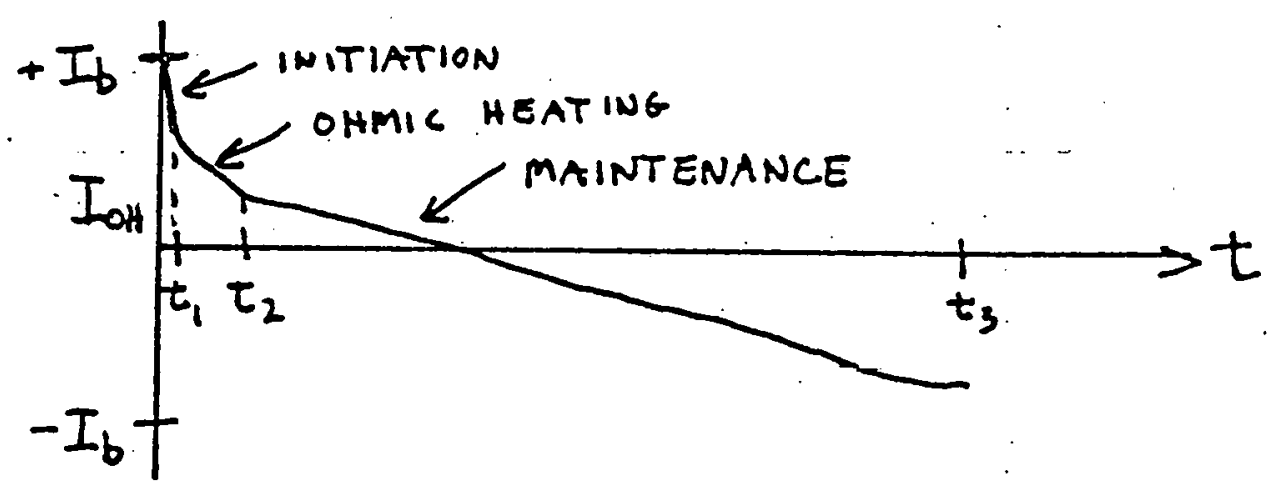

Figure 8. I ohve. time for a typical cycle.

The coil is initially charged up (biased) to a current of $+I_{b}$ and held at this value while the cold gas is pumped into the vacuum vessel. The current is then allowed to drop rapidly for a short period, inducing a large electric field in the plasma for initiation. The coil is then connected to an energy storage sink which resonantly allows the coil current to decrease and reverse, finally reaching the value $-I_{b}$ at the end 
of the cycle. The monotonically decreasing coil current thus produces a positive induced plasma voltage throughout the cycle. At the end of the cycle, $t_{3}$ above, the necessary circuitry is provided to allow the current to again reverse and return to $+I_{b}$ to start another cycle. Some calculations have shown (26) however, that such an ideal cycle is not attainable and that as much as $50 \%$ of the initial stored energy will be resistively dissipated in the cycle. One purpose of the present study is to determine how much of this loss will be incurred during the initiation pulse.

A canonical form of the circuitry necessary to achieve the above current waveform is shown in Figure 9.

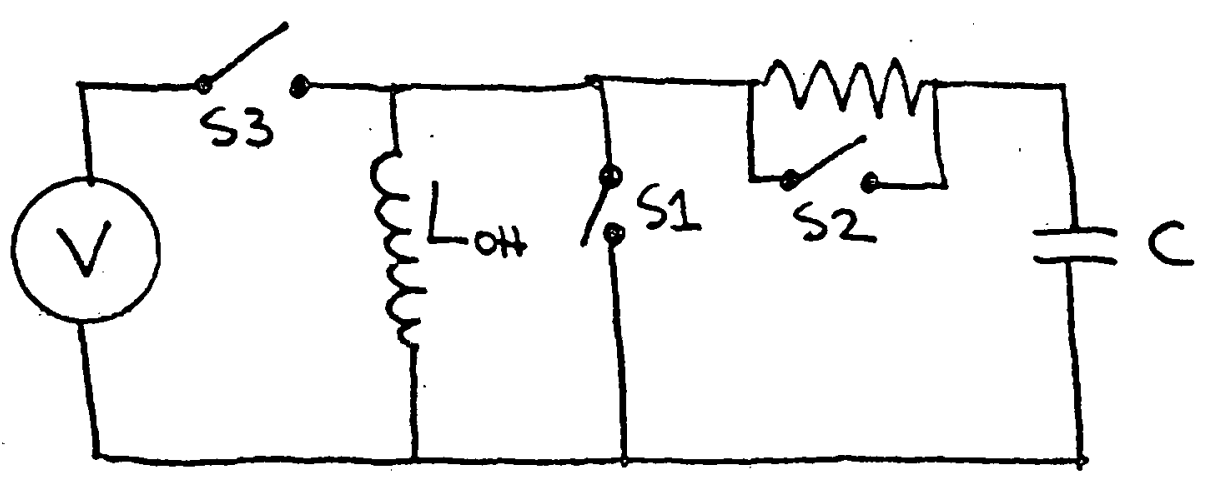

Figure 9. Canoncical OH drive circuit

$V$ represents the primary energy store, which initially charges up $I_{\mathrm{OH}}$ to $+I_{b}$ by closing $\$ 3$ prior 
to operation. Then $S I$ is closed, shorting $L_{\mathrm{OH}}$ and maintaining $I_{0} a t+I_{b}$ until the start of the cycle, at which time $S I$ is opened. Ioh then begins to discharge through $R I$ and $C$ at a rapid rate, generating the initiation voltage in the plasma. At the end of the initiation pulse, $R I$ is shorted by $S 2$ and the current continues to decrease at the slower rate required to aditionally heat and finally maintain the plasma throughout the burn cycle.

After performing our initial physics calculations, it was found desirable to add an additional section to the drive circuit for the purpose of initially boosting the ionization voltage. The resulting circuit is the primary configuration utilized in the final calculations and is shown below in Figure 10 .

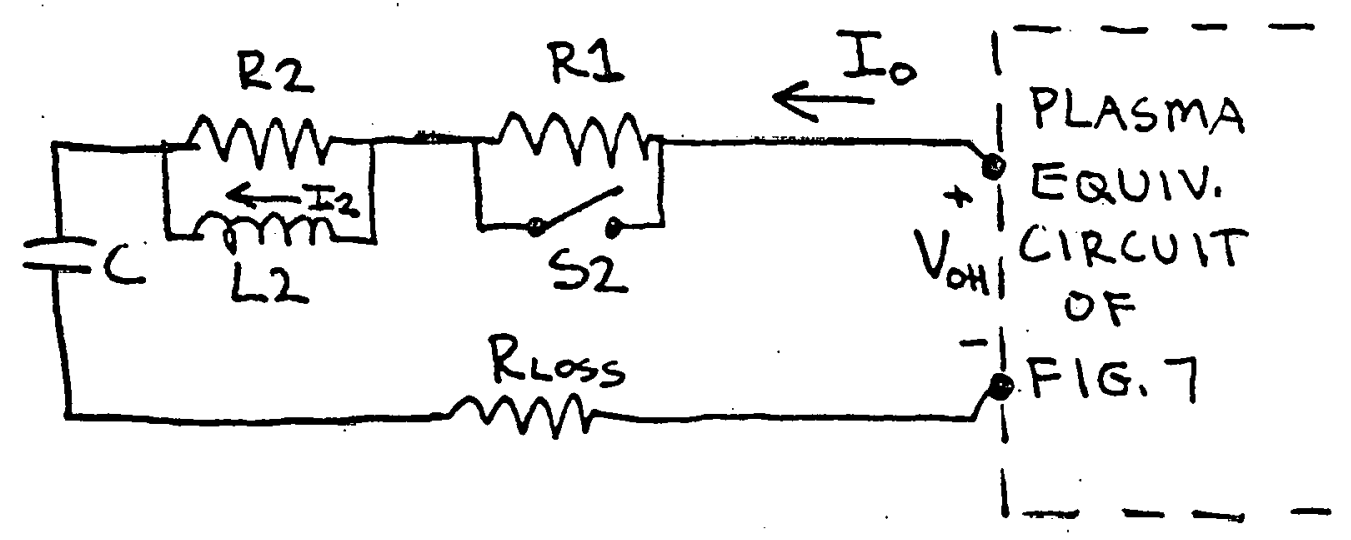

Figure 10. Complete equivalent $O H$ drive circuit. 
$R_{L}$ represents the circuit losses. The net loop voltage equation for this circuit results in:

$V_{O H}=I_{0}\left(R_{1}+R_{2}\right)-R_{2} I_{2}+\frac{1}{C} \int_{0}^{t} I_{0} d t$

with the auxiliary equation

$$
\dot{I}_{2}=\frac{R_{2}}{L_{2}}\left[I_{0}-I_{2}\right]
$$

Substituting (9-1) into the last of equation set $(8-13)$ we obtain

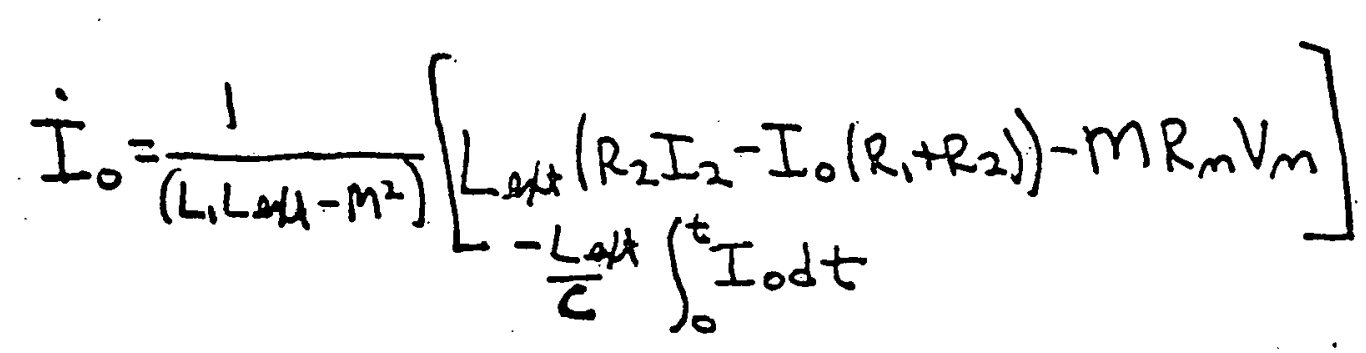

Thus, we have arrived at the final formulation for the problem, which consists of the set of equations for the n plasma shell currents, the of drive circuit currents and the plasma rate equations which are collected together below for reference. 
48

COMPLETE RATE EQUATION SET

$$
\begin{aligned}
& \frac{d M_{e}}{d t}=M_{e} M_{0} R_{1}-M_{e} M_{i} R_{2}+\sum_{i=1}^{8} S_{i} O_{i}-\frac{M_{e}}{T_{p}} \\
& \frac{d M_{i}}{d t}=M_{e} M_{0} R_{1}-M_{e} M_{i} R_{2}-\frac{M_{i}}{\tau_{p}} \\
& \frac{d M_{0}}{d t}=\Gamma-M_{e} M_{0} R_{1}+M_{e} M_{i} R_{2} \\
& \frac{d O_{1}}{d t}=-M_{e} O_{1} S_{1} \\
& \frac{d O_{2}}{d t}=M_{e} O_{1} S_{1}-M_{e} O_{2} S_{2} \\
& \frac{d O_{3}}{d t}=M_{e} O_{2} S_{2}-M_{e} O_{3} S_{3} \\
& 1 \\
& \frac{1}{1} \\
& \frac{d O_{8}}{d t}=M_{e} O_{7} S_{7}-M_{e} O_{8} S_{8} \\
& \frac{3}{2} \frac{d}{d t}\left(M_{i} T_{i}\right)=Q_{e i}-\frac{3}{2} \frac{m_{i} T_{i}}{\tau_{E i}}
\end{aligned}
$$


49

$$
\begin{aligned}
& \frac{3}{2} \frac{d}{d t}\left(M_{e} T_{e}\right)=\sum_{i=1}^{M} I_{i}^{2} R_{i}-Q_{e i}-M_{e} M_{0} R_{1} \varphi_{H} \\
& -M_{e} M_{i} P_{j}-M_{e} \sum_{i=1}^{8}\left[O_{i} S_{i} \varphi_{i}+P_{i} O_{i}\right] \\
& -\frac{3}{2} \frac{M_{e} T_{e}}{T_{E_{e}}} \\
& \frac{d I_{1}}{d t}=\frac{R_{2}}{L_{2}}\left[I_{2}-\frac{R_{1}}{R_{2}} I_{1}\right] \\
& \frac{d I_{2}}{d t}=\frac{R_{3}}{L_{3}}\left[I_{3}-\frac{R_{2}}{R_{3}} I_{2}\right]-\frac{d I_{1}}{d t} \\
& \frac{d I_{m-1}}{d t}=\frac{R_{m}}{L_{m}}\left[I_{m}-\frac{R_{m-1}}{R_{m}} I_{m-1}\right]-\frac{d I_{m-2}}{d t} \\
& \frac{d I_{m}}{d t}=-\frac{R_{m}}{L_{m}}\left(1+\frac{L_{m}}{L_{\text {opt }}}\right) I_{m}-\frac{m}{L_{\text {oft }}} \dot{I}_{0}+\frac{R_{m-1}}{L_{m}} I_{m-1} \\
& \begin{array}{l}
\frac{d I_{0}}{d t}=\frac{1}{\left(L_{1} \operatorname{Led}-M^{2}\right)}\left[\begin{array}{l}
\left.L \text { ext }\left(R_{2} I_{2}-I_{0}\left(R_{1}+R_{2}\right)\right)-M R_{m} V_{m}\right] \\
-\frac{L e d t}{c} \int_{0}^{t} I_{0} d t
\end{array}\right. \\
\frac{d I_{2}}{d t}=R_{2}
\end{array} \\
& \frac{d I_{2}}{d t}=\frac{R_{2}}{L_{2}}\left[I_{0}-I_{2}\right]
\end{aligned}
$$


IV. RESULTS

\section{Initial Conditions}

The initial conditions utilized in these simulations are supposed to be represe: -ative of typical TFTR experiments in which a reduced rac:us plasma is formed at a slightly increased major radius of $350 \mathrm{~cm}$. and grown off of the limiter by vertical field control combined with controlled gas flow. During the initiation phase the particle and energy confinement times are uncertain, so that we have utilized the value $\tau=20$. msec. as suggested by Hawryluk (29).

The plasma is assumed to be initially lonlzed to a small degree by some RF heating means, typically .18. The initial temperatures used are $\mathrm{T}_{\mathrm{e}}=\mathrm{T}_{\mathrm{i}}=1 \mathrm{eV}$. The results are very insensitive to these assumed values:

We have performed two types of simulations for the purpose of (A) evaluating basic physical parameters relevant to ionization and radiation losses, and (B) determining electrical requirements of the initiation. process. For the physics simulations the simplified formulation of Chapter II was utilized. For the electrical simulations the full formalism of chapter III 
was utilized, where it is noted that the latter required considerably more computation time due to the increased number of equations and the shorter time constants involved. In fact, the electrical calculations were performed after the physics code was found to be fully operational.

\section{Physics Results}

The physics related simulations, performed primarily to permit a comparison between the physical models used in the study with those in the references cited earlier, utilized the same set of parameters as in the work of reference 2 :

$$
\begin{aligned}
& n_{e}=1 \times 10^{11} \mathrm{~cm}^{-3} \\
& n_{0}=3.5 \times 10^{13} \mathrm{~cm}^{-3} \\
& n_{0 x}=0,3, \text { and } 6 \times 10^{11} \mathrm{~cm}^{-3}
\end{aligned}
$$

where the listed values for Oxygen correspond to 1mpurity concentrations of 0,18 , and 28 of the final plasma density of $3 \times 10^{13}$. Various values of loop voltage between 50 and 200 volts were applied to the plasma. In Figure 11 the value of the ionization rate co-efficient for $\mathrm{H}^{0}$ is plotted, in which the values shown are normalized to a value for $\mathrm{M}_{\mathrm{e}} \mathrm{M}_{\mathrm{H}}=10^{26}$. (Much of the literature on atomic processes in plasmas in this temperature range has utilized this reference 


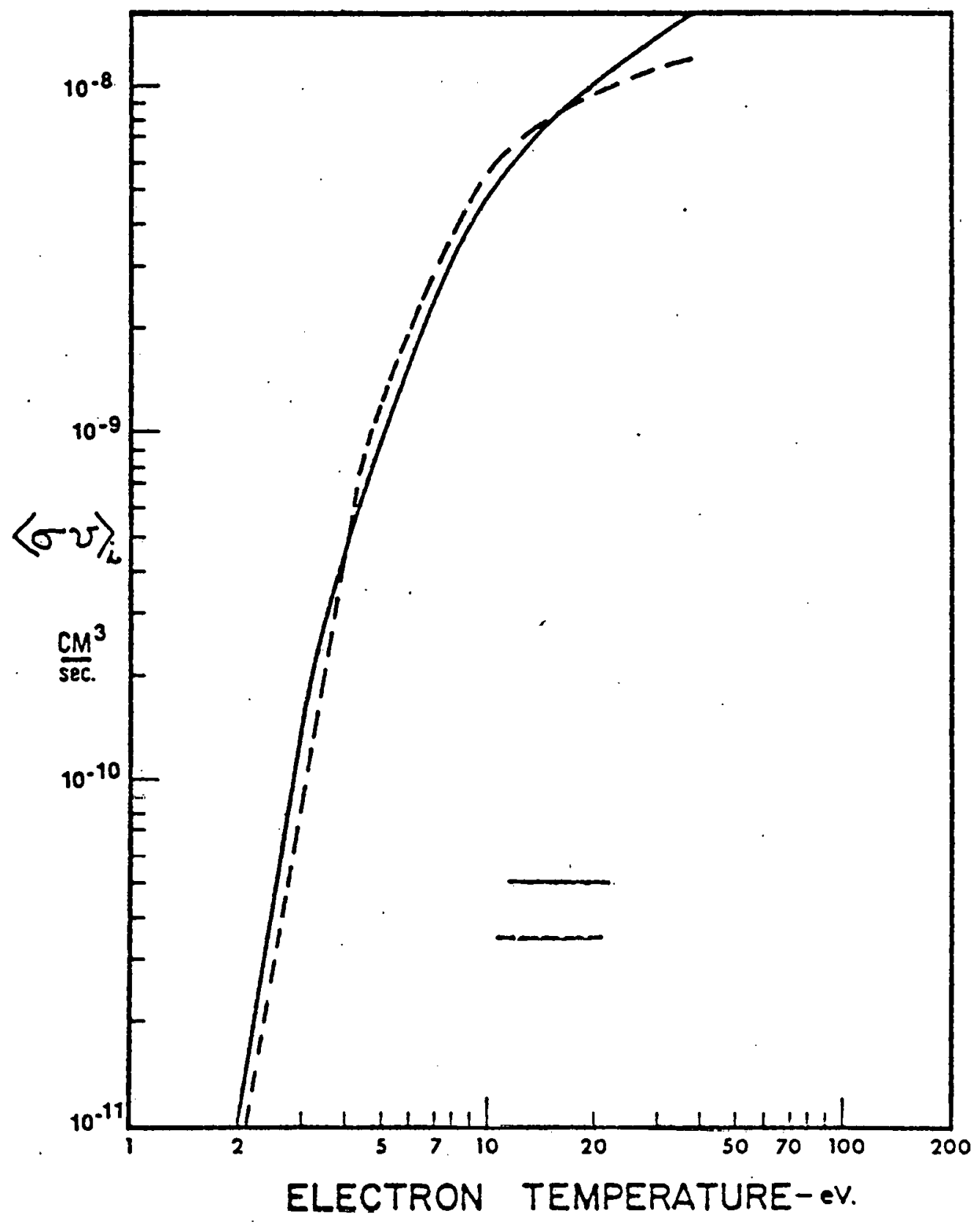

Figure 11. $\mathrm{H}^{0}$ ionization rate vs. electron temperature. Neutral gas is assumed to have $\mathrm{T}_{\mathrm{H}}=0$. 
value, a historical convention which will also be observed here for convenience in checking our present results.) Note that the data in this graph is not the result of our computations, but is simply a display of the original data of Lotz, displayed here for completeness.

In Figure 13 the absolute magnitudes of the densities of all Oxygen ionization states is plotted vs. temperature for a typical case. We have found the shapes of these curves to be quite consistent in the various runs performed, with the exception of the values found for $0_{I I}$. The value for the latter is found to be quite dependent on the gross Hydrogen ionization rate, which is strongly dependent on the applied voltage. This is not surprising considering that the ionization energies for these two atoms, $\mathrm{H}^{\circ}$ and $O_{I}$ are nearly identical. We have also made a comparison between the values of Figure 13 and a crude solution of an 8-level set of Saha equations for this process, with the expected result that the latter grossly overestimates the concentrations.

We have also found a great difference between the results in Figure 13 and the (coronal equilibrium) calculations of Cox and Tucker (10), particularly the latter's consistent overestimate of the higher states, at any temperature. 


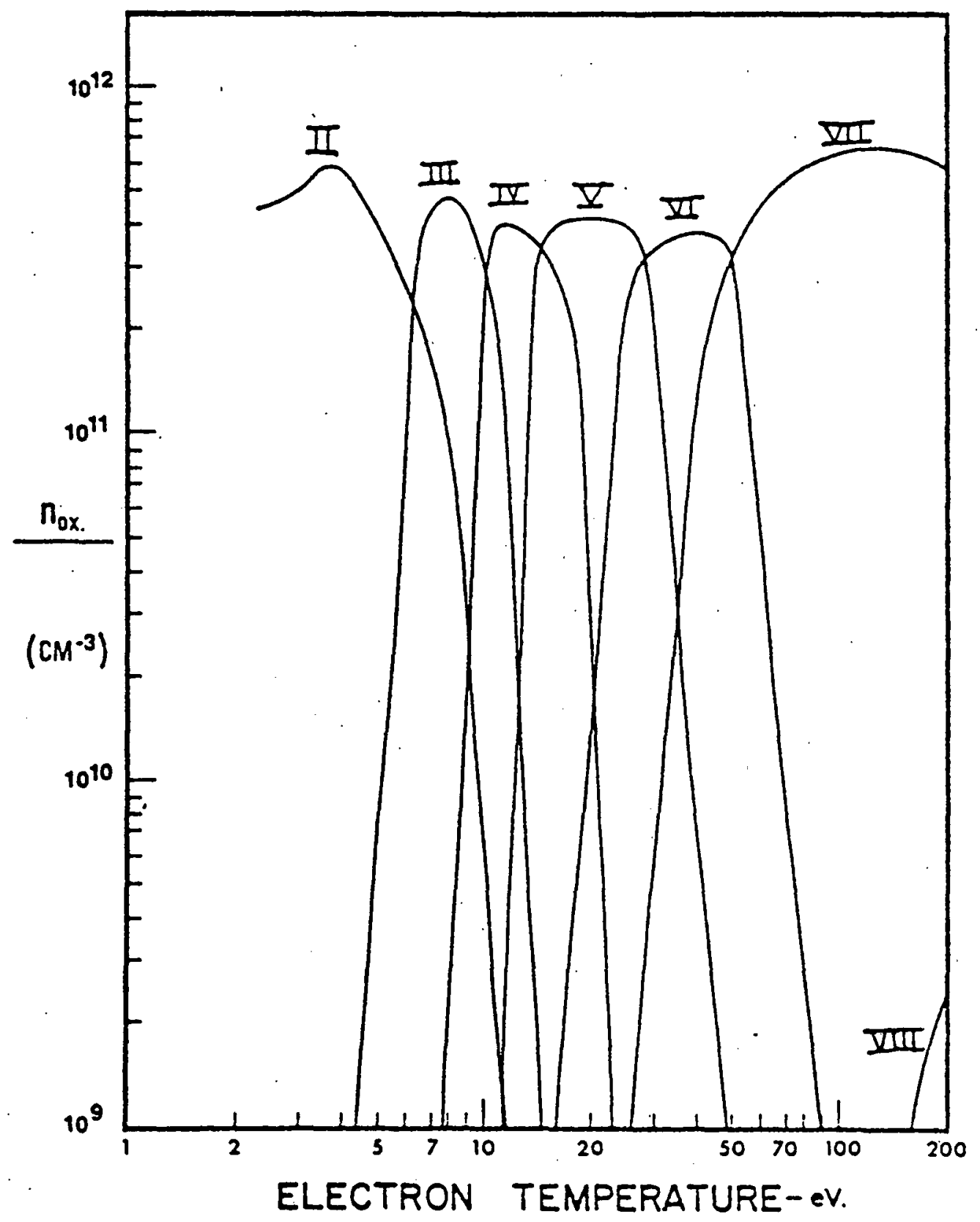

Figure 13. Absolute densities of atoms in various ionization states. Total oxygen density is $6 \times 1011 \mathrm{~cm} .-3$. 
The results of the oxygen line radiation calculations are presented in Figure 12. These results are a central feature of this study, so the corresponding data from the previously cited references are also plotted for accurate comparison. We note that with respect to the results of Hawryluk and schmidt, there is some confusion. Their results reproduced here from their Figure 1, do not explicitly state the origin of the data; i.e., is it the results of their computations or simply a re-listing of the original data from Duchs and Griem? Their data also would tend to indicate that above an electron temperature of $40 \mathrm{eV}$, all of the Oxygen is ionized, with perhaps the exception of ${ }^{0} \mathrm{VIII}$. Below this temperature range, the present results are in excellent agreement.

The DPR data, on the other hand, shows a considerable deviation from ours, by almost an order of magnitude in the important central temperature range. As this temperature range is of some importance in the early stages of ohmic heating, we feel that our data, and the sizeable correction that it represents, should be considered the more relevant for future studies in this temperature range. 


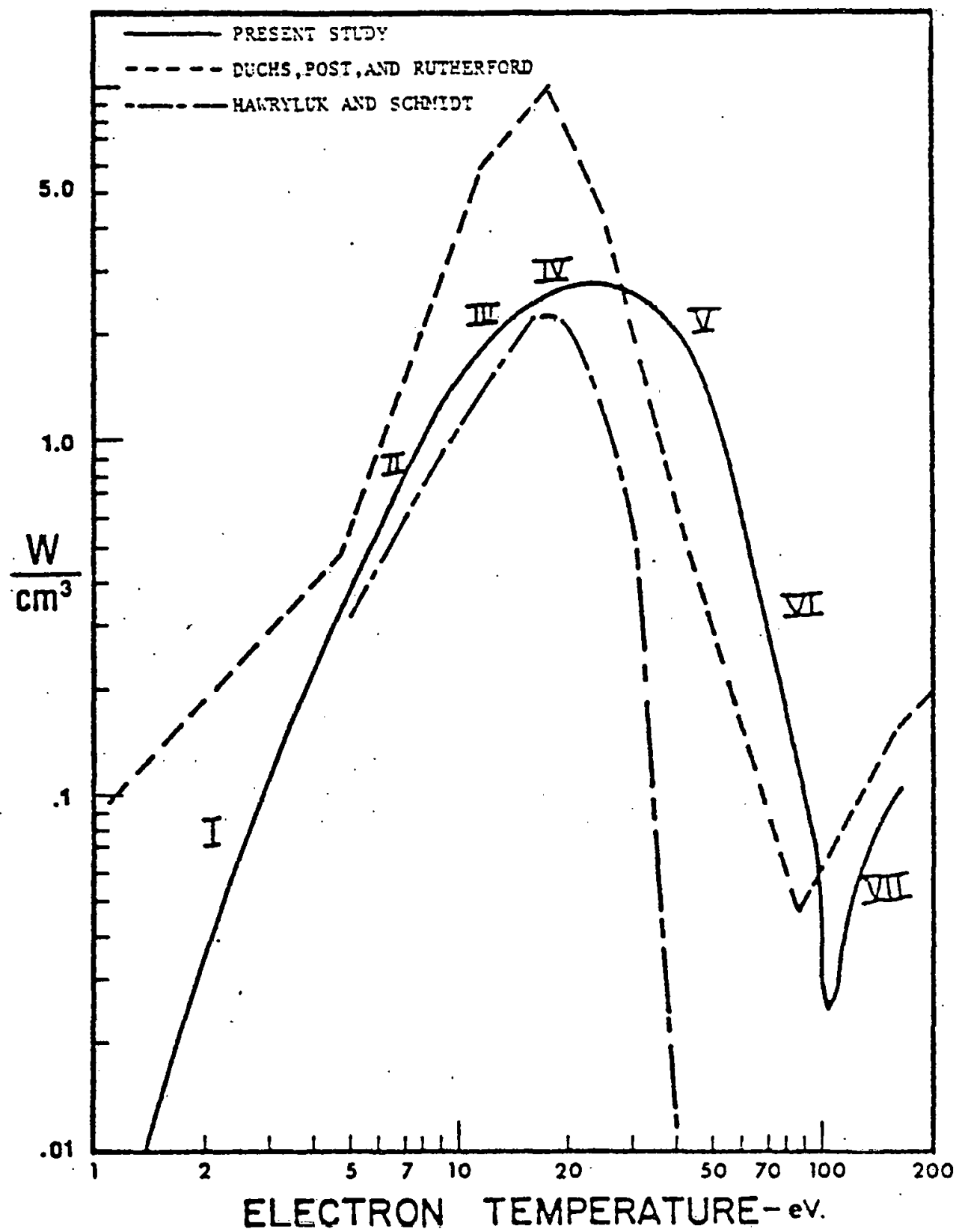

Figure 12. Power loss density due to oxygen line radiation; normalized to a density product $n \mathrm{n}^{\mathrm{n}}=10^{26} \mathrm{~cm}^{-6}$.
Ionization numbers represent state which is the predominant contributor to the total power. 
In Figure 14 we present the calculated value of $\langle z\rangle$ which is defined as:

$\cdot\langle z\rangle_{\alpha x}=\sum_{i} m_{i} \partial_{i} / \sum_{i} m_{i} \quad i=I, I$, III, $\cdots$ IX

As in the reported results of DPR, no significant difference was observed between the calculated values of $\langle z\rangle^{2}$ and $\left\langle z^{2}\right\rangle$. This simply reflects the fact that the ionization energies of the various oxygen states are so widely separated that at any given temperature, one state is predominantely abundant.

Figure 14 also shows the total power going into the ionization of oxygen, with the reference normalization used. The deep well in this curve at $100 \mathrm{ev}$. occurs because of the great difference in ionization potential of the states $0_{\mathrm{VII}}$ and $\mathrm{o}_{\mathrm{VIII}}$. From this curve the following fact can be inferred: for studies of ohmic heating and related processes in the .1-1 kev. temperature range, the oxygen present can be considered to be fully ionized to the VII-state (this has the computational advantage of eliminating the rate equations for the first six ionization states).

Figures 15 thru 17 present the results of the calculations of plasma current and temperature evolution for a system driven by the complete $\mathrm{OH}$ circuit of Figure 10. These results are in excellent agreement with those of Hawryluk and schmidt (2), particularly 


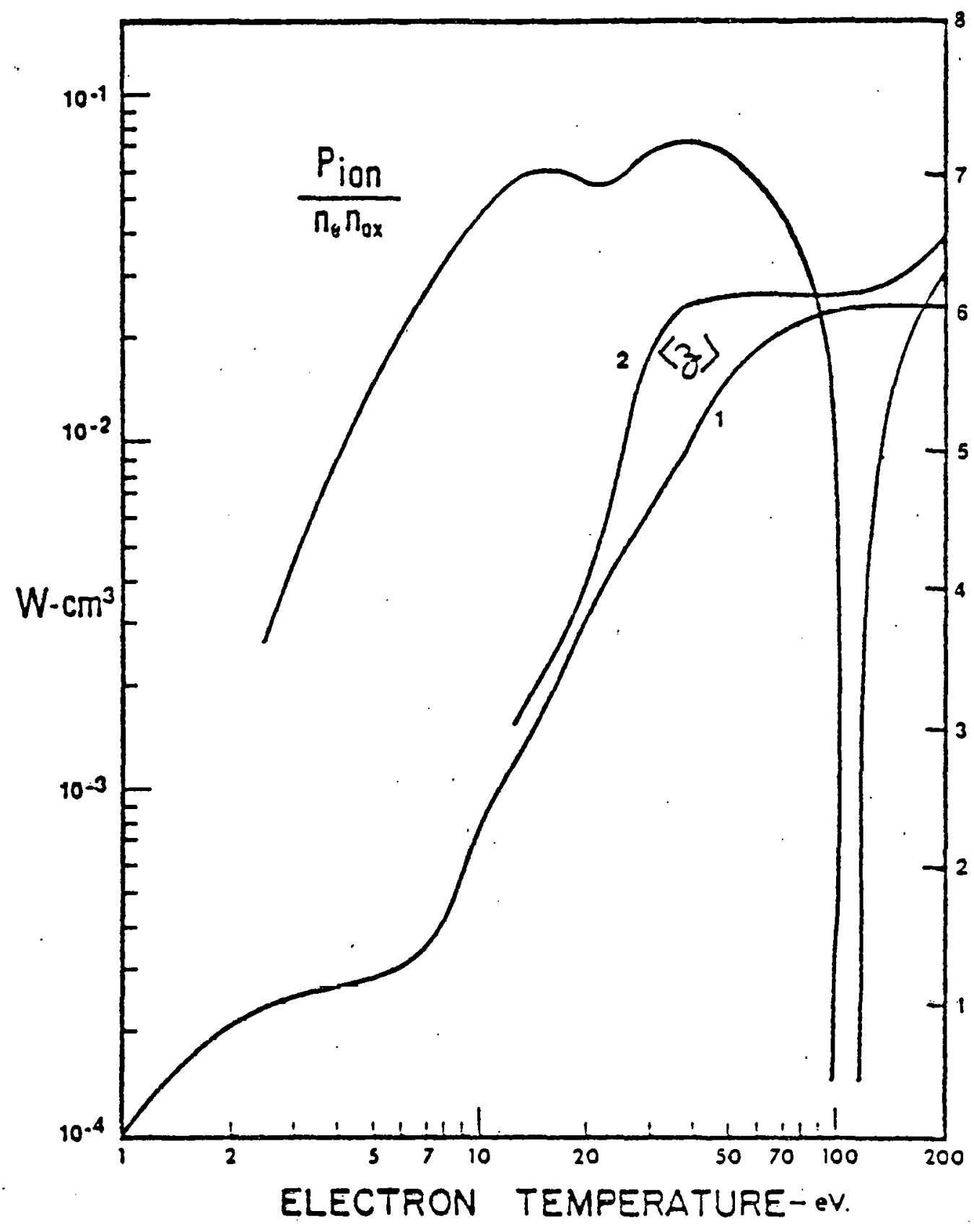

Figure 14. Power flow into oxygen ionization, normalized to $\mathrm{n}_{e^{\mathrm{n}}}=1026 \mathrm{~cm}$. -6 mean charge $z$ of oxygen population is also shown. 
PLASMA CURRENT - $k$ A.

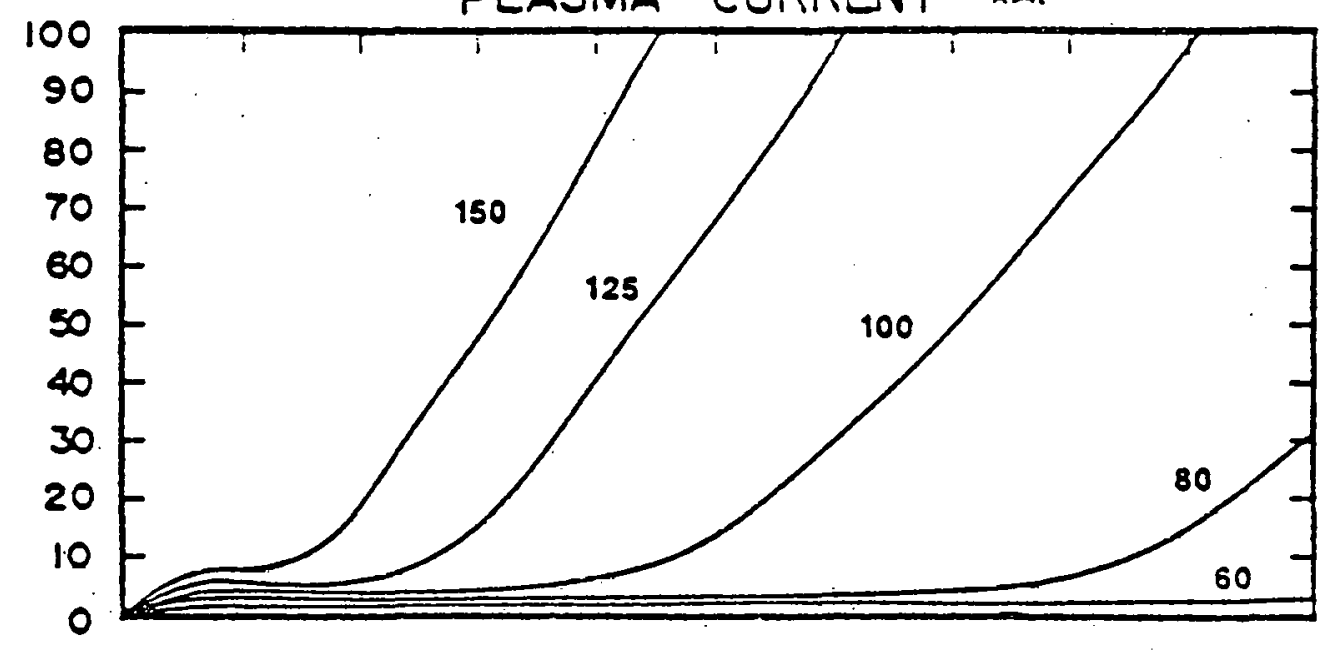

ELECTRON TEMPERATURE- $-V$.

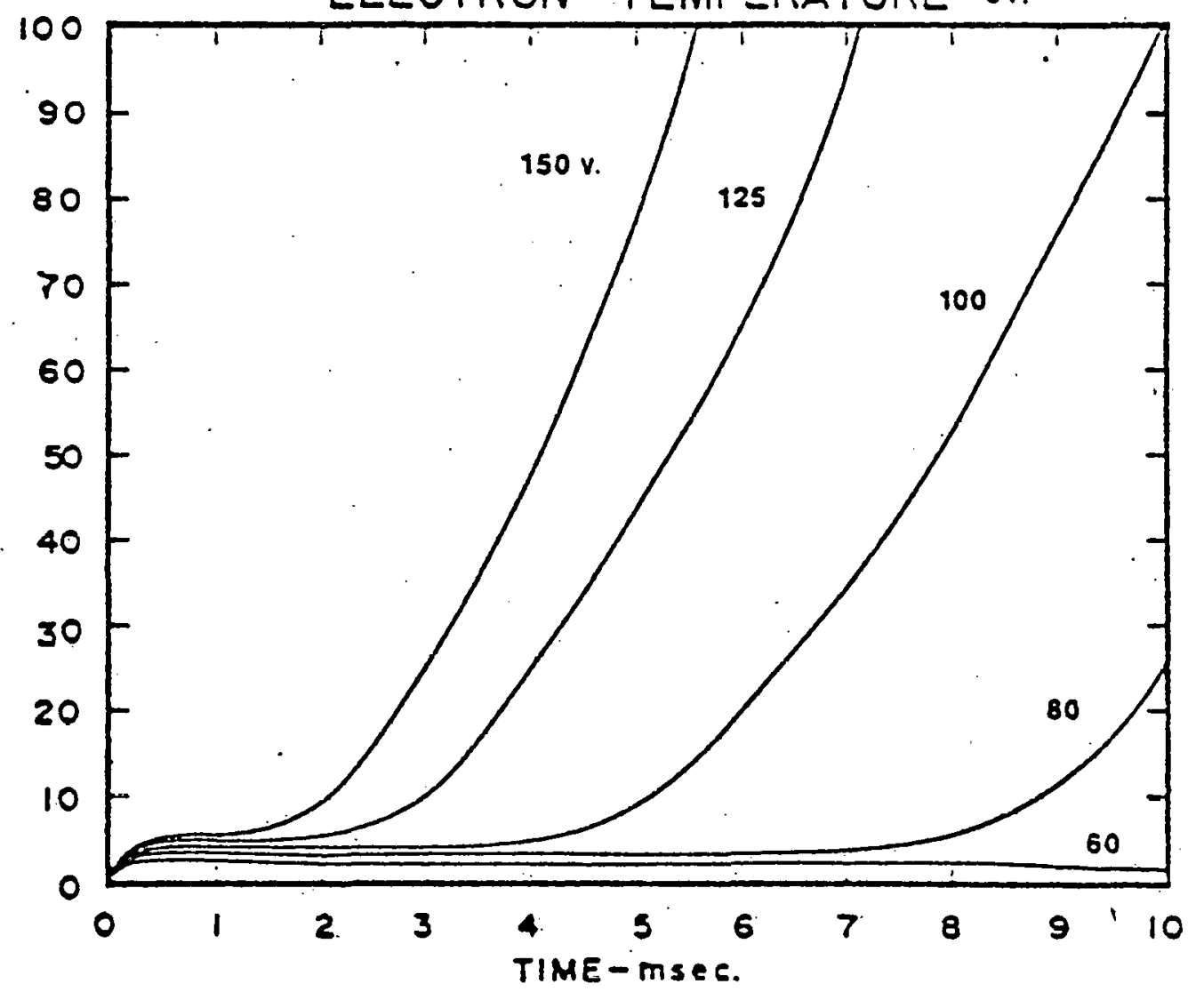

Figure 15. Temperature and current evolution in a plasma containing no impurities for various applied loop voltages. 
PLASMA CURRENT - $k$ A.

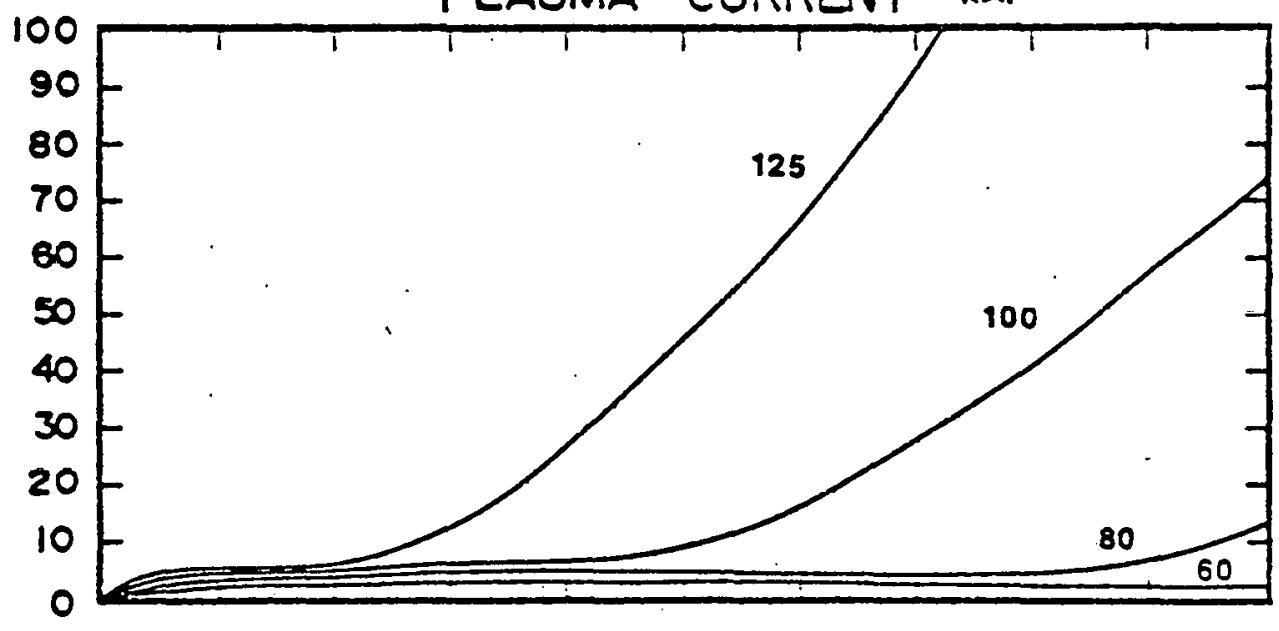

ELECTRON TEMPERATURE- eV.

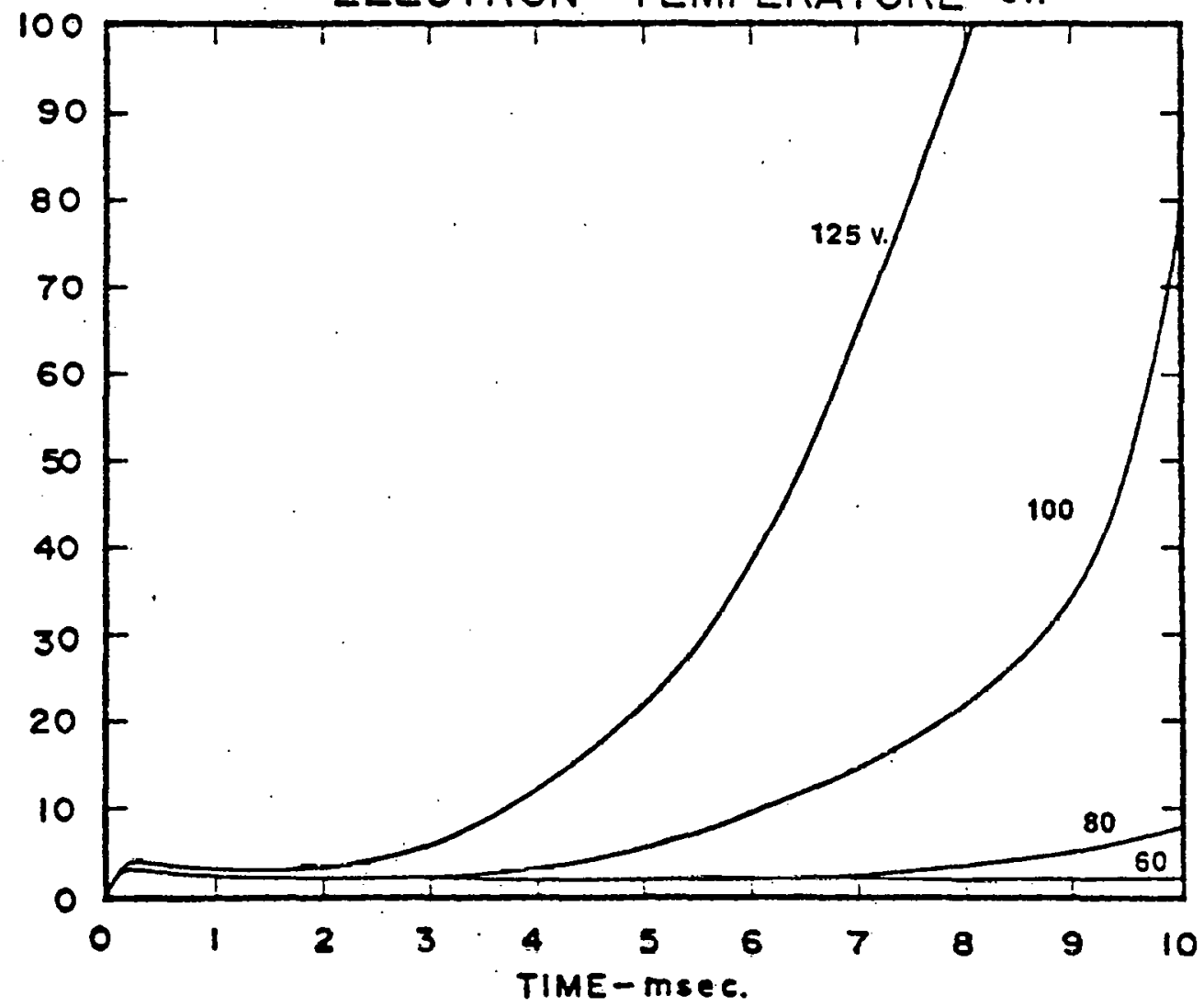

Figure 16. Temperature and current evolution in a plasma containing is oxygen impurity concentration. 

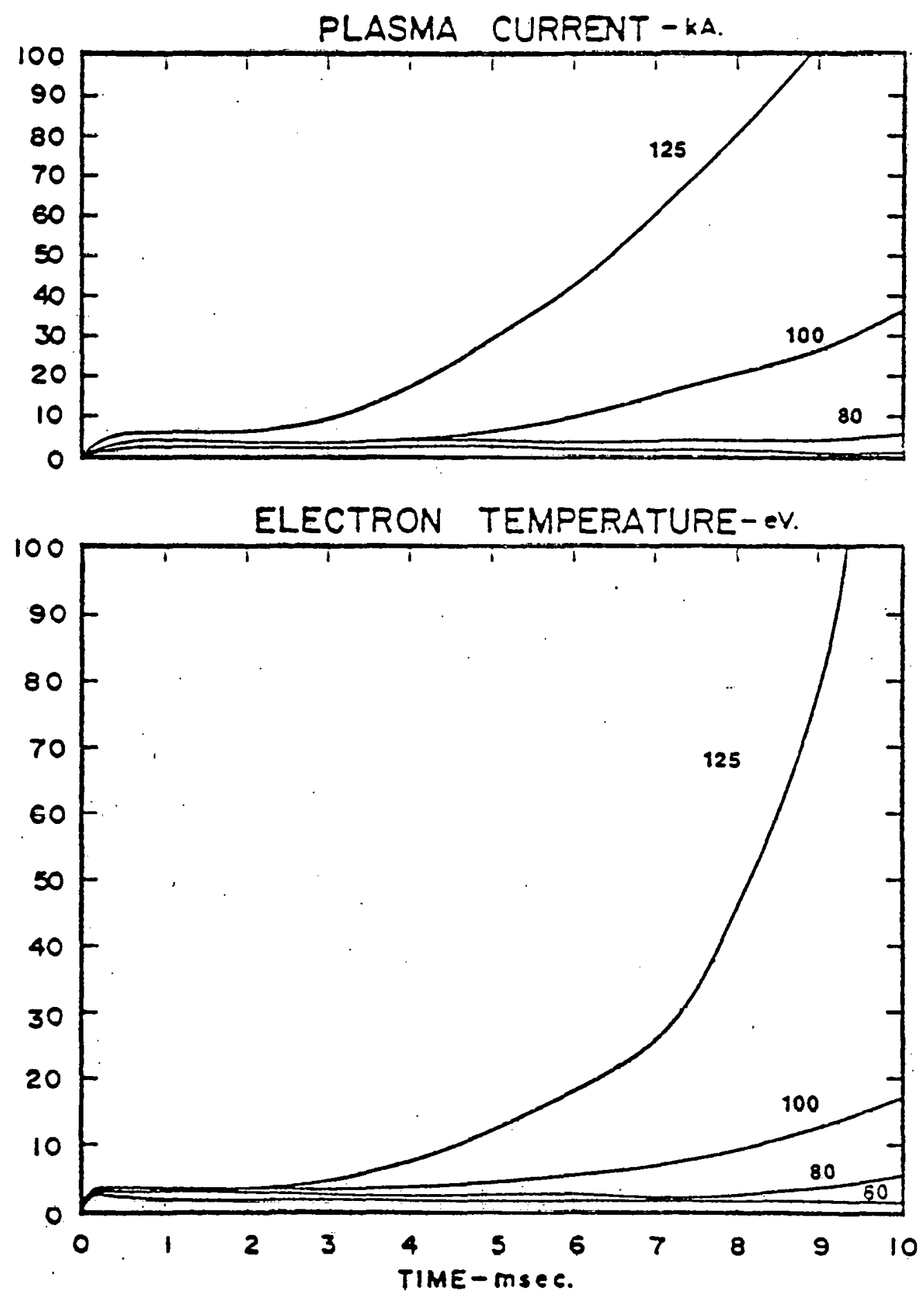

Figure 17. Temperature and current evolution in a plasma containing $2 \%$ oxygen impurity concentration. 
with regard to the existence of a lower voltage threshold for satisfactory plasma evolution. Our results seem to be somewhat more optimistic with regard to the final values of temperature and current attained at. $t=10$ msec. This is perhaps due to the improved calculations for the line radiation loss employed here, which was seen previously to result in a lower loss rate than in previous calculations.

Finally, as an aid in the understanding of the relative importance of the various physical processes which affect the transient plasma formation, we present in Figure 18 a plot of the gross power density of the major power sources and sinks as the discharge evolves. $P_{\mathrm{OH}}$ is the total resistive electrical power input to the plasma by $O H$ induction, $P_{\text {line }}$ is the amount of power dissipated by line radiation, $P_{i o n}$ is the power used to ionize atoms, and $Q_{e i}$ is the power flow from electrons to ions (collisional ion heating). The run selected for this graph represents a moderately rapid heating scenario.

\section{Electrical Results}

A. Current and Temperature Evolution

In planning the calculations for this section, several goals were identlfled at the oulset, 


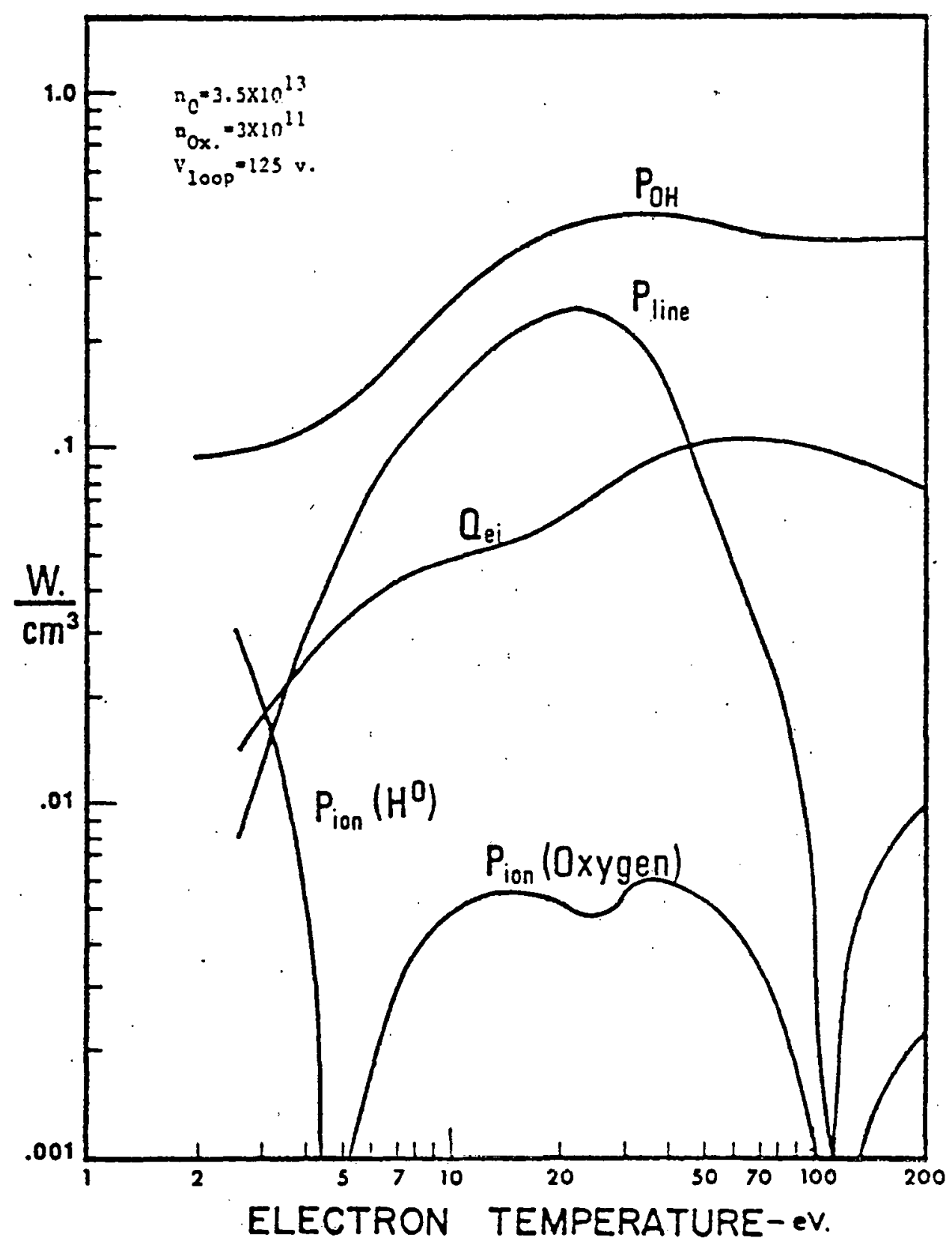

Figure 18. Power flows to various processes in and evolving plasma. $P_{\text {oh }}=$ total power input to plasma, $\mathrm{P}_{\text {line }}=\mathrm{line}$ radiation lose, $P_{\text {ion }}=$ ionipation power loss, $Q_{e i}=$ electron-ion power transfer. 
which are stated in the form of the following questions :

1. Is there any freedom in selecting the electrical parameters in such a way as to most efficiently heat and ionize the plasma?

2. How much can the required heating energy be minimized by optimal choice of parameters in the ohmic heating system?

3. Are the optimal electrical parameters within the available technological limits?

4. What is the predominant physical limitation during initiation?

After analyzing the results of the previous section, we observed an interesting fact about the evolution of the plasma: successful evolution is preceeded by an initial peak in the electron temperature, followed by a decrease to a plateau region lasting for several msee. During the platean, little heating (i.e., $\Delta T_{e}$ ) takes place and most of the available energy goes into $\mathrm{H}^{\circ}$ ionization. We then found, empirically, that a boost in the applied voltage during the first msec. of the discharge was quite effective in shortening the plateau period, and the subsequent period of final ionization and ohmic heating proceeded much more rapidy. A detailed analysis revealed further, that the energy stored both in the thermal motion of the electrons and in the self-inductance field of the electron current were enhanced by the boost, providing a sort of 
"thermal inertia" to aid in going through the initial ionization phase.

It was this reasoning that led to the implementation of the boost circuit employed in the final $O H$ drive circuit of Figure 10. Initially, the concept was tested by placing an additional resistance in the $\mathrm{OH}$ coil discharge circuit at $t=0$ and switching it out after about one msec. The technical problems of such a switching action, however, are quite severe as the required switch would be required to interrupt a circuit carrying about $40, \mathrm{kA}$. at an open-circuit blocking voltage of 20-50 kV. Hence, the use of a fairly simple inductance was found to produce the same effect without the associated switching problems.

In our final calculations, the following base set of parameters was selected:

TABLE 3

Io

L2/R2

$R_{1}$

no

ne

nox

$c$
$40 \mathrm{kA}$.

$1 \mathrm{msec}$.

.3-1.0 ohms

$3.5 \times 10^{13} \mathrm{~cm}^{-3}$

$1 \times 10^{11} \mathrm{~cm}^{-3}(.38)$

$3 \times 10^{11} \mathrm{~cm} .^{-3}$

$1 \mathrm{fd}$. 
The range of $R_{1}$ selected produces a peak plasma voltage in the range of 60-200 volts, the same range as used in the previous section. The value of $C$ specified produces a full flux-reversal time of $\sim .5$ sec.

The plasma was represented as a 10-section, uniform current density diffusive line as described previously (i.e., ten current shells of equal thickness). Figure 19 presents the results for nonboosted initiations with values of $R_{1}$ selected to produce peak values of plasma voltage of $70,90,120$, 135, and 165 volts. These are similar to the values used in the previous section and the results are.. essentially identical (compare Figure 16). "An expanded view of the first millisecond of the discharge is shown in Figure 20, in which the initial temperature peak is clearly visible.

To test the operation of the boost circuit, a number of cases were tried utilizing the parameters shown in Table 4 where $R_{1}, R_{2}$ and $I_{2}$ are defined in Figure 10. $v_{1}$ is the peak voltage which appears across the $\mathrm{OH}$ coil terminals, a quantity of engineering concern. $v_{p}$ is the peak voltage induced in the plasma and is numerically equal to $v_{n}$ in the analogue circuit of Figure 7 .

In this series of calculations, the total resistive energy used in the discharge was calculated 
PLASMA CURRENT - $x$ A.

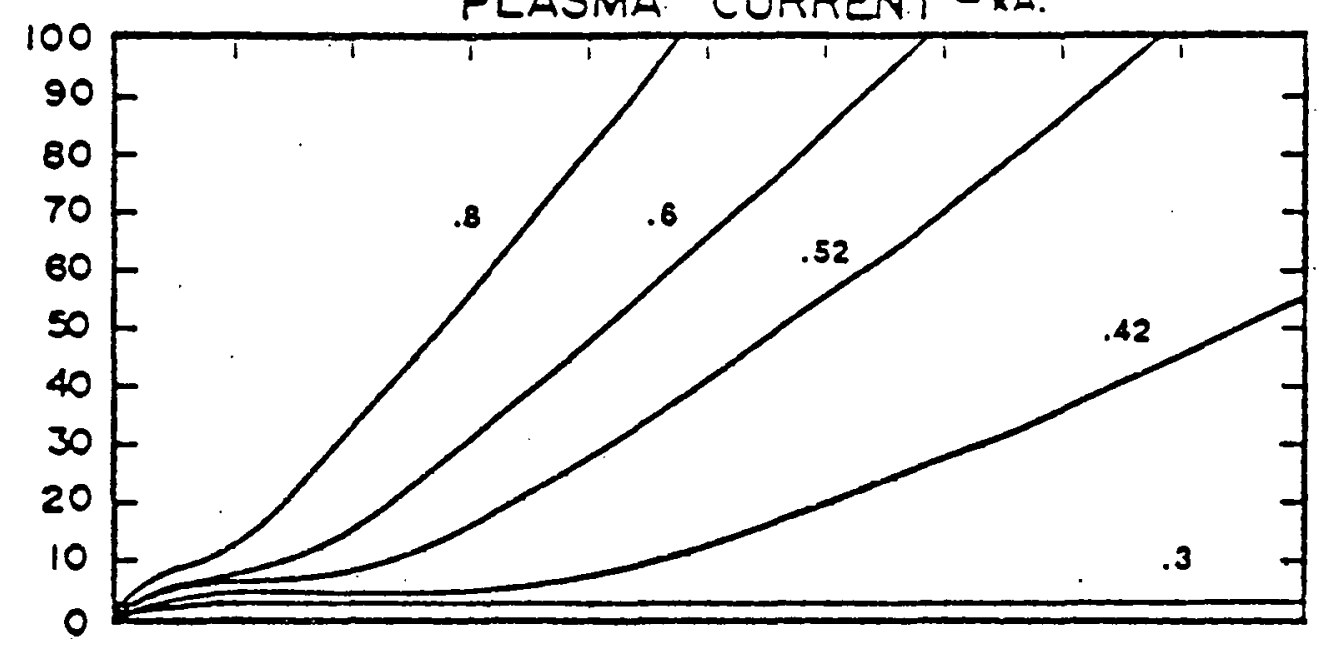

ELECTRON TEMPERATURE- $\mathrm{eV}$.

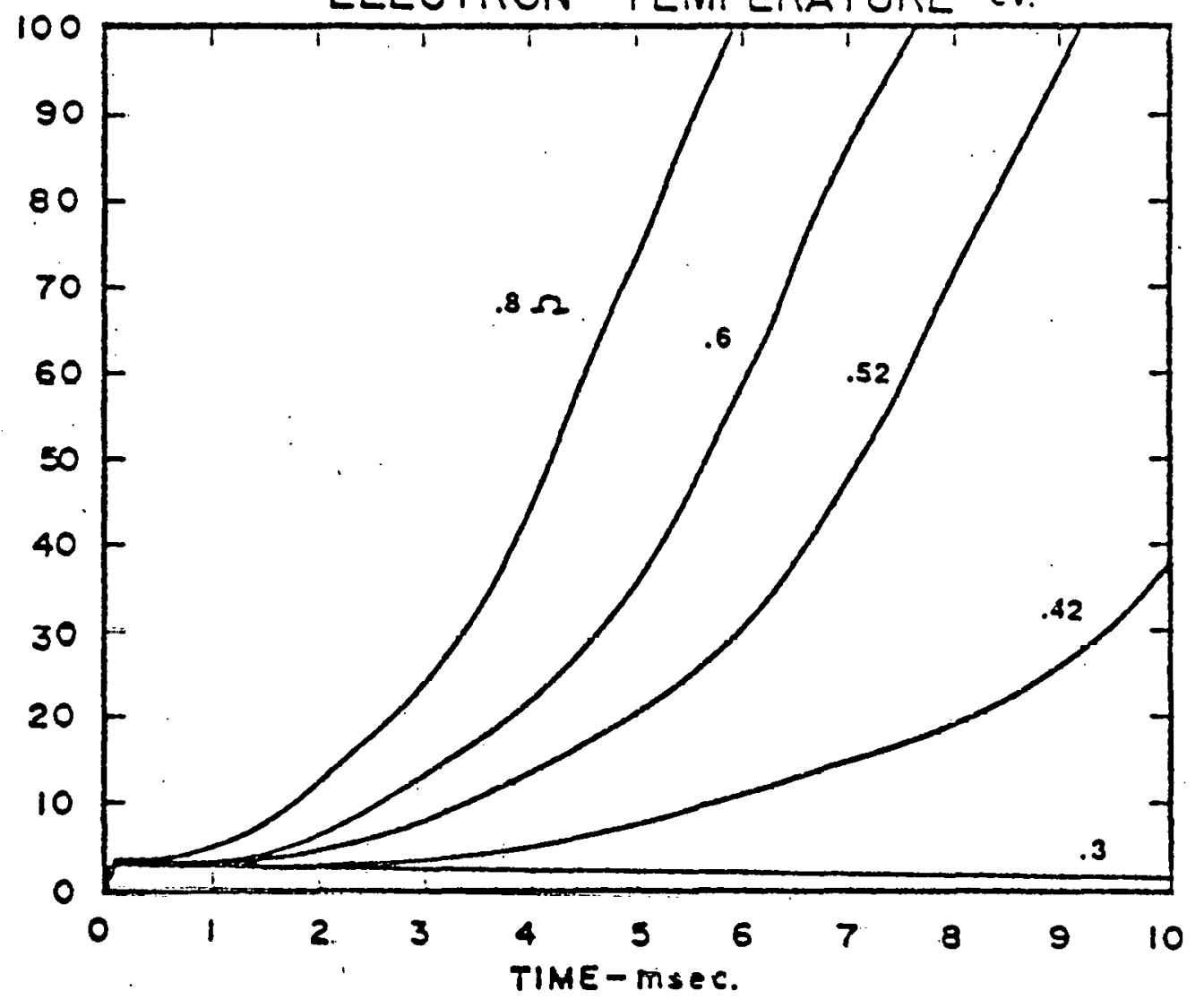

Figure 19. Plasma electron temperature and current evolution for various values of initiation resistance $R_{1}$ and parameters of rable 3 and. Table 4. 
PLASMA CURRENT - $k A$.

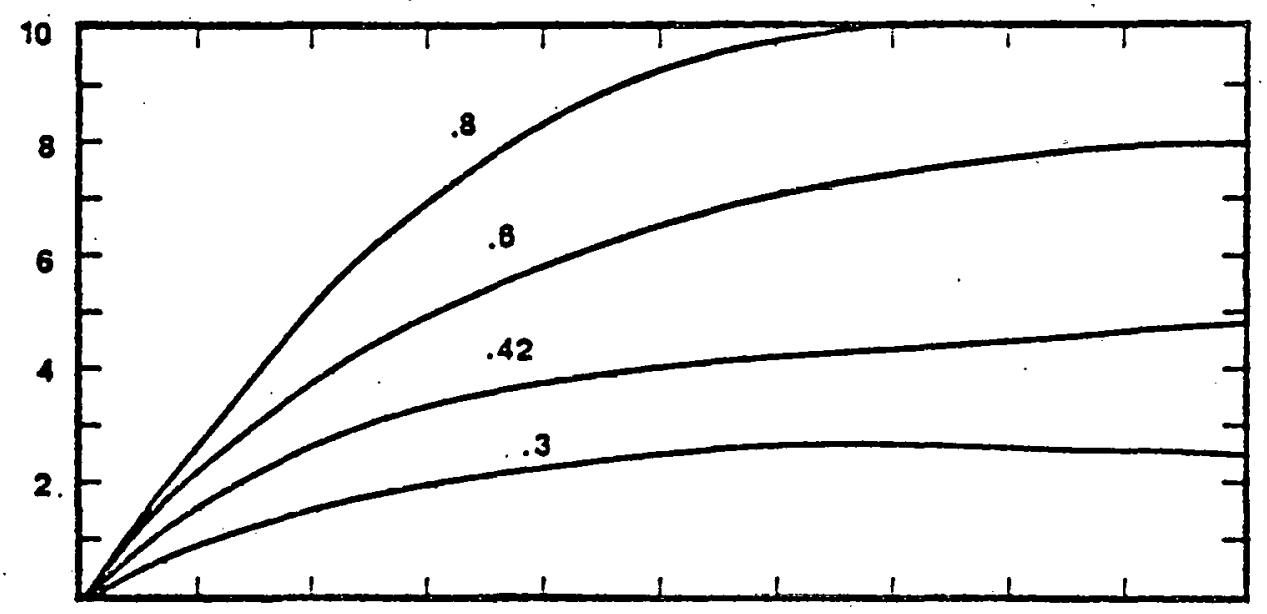

ELECTRON TEMPERATURE- $\mathrm{eV}$.

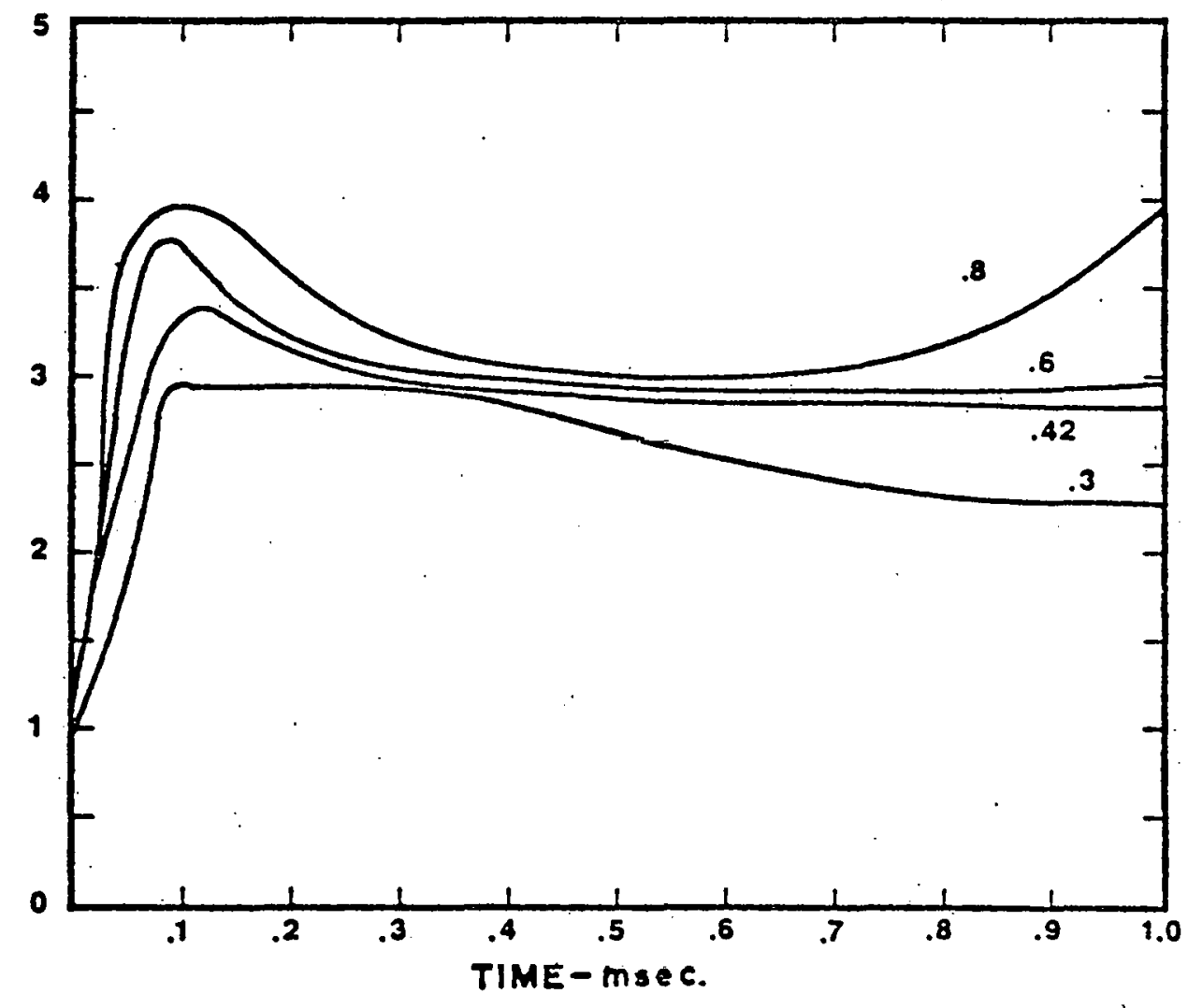

Figure 20. Enlarged view of first millisecond of discharges presented in Figure 19 . 
TABLE 4

\begin{tabular}{cccccc}
\hline $\begin{array}{c}\text { Case } \\
\text { No. }\end{array}$ & $R_{I}$ & $R_{2}$ & $I_{2}$ & $V_{1} \max$ & $V_{P} \max$ \\
\hline 1 & .52 & 0 & - & $20.8 \mathrm{kV}$. & $120 \mathrm{~V} \cdot$ \\
2 & .40 & 1.0 & .0005 & 56 & 177 \\
3 & .75 & 1.5 & .0007 & 90 & 218 \\
4 & .30 & 1.2 & .0007 & 60 & 183 \\
5 & .52 & 1.0 & .0005 & 60.8 & 186 \\
6 & .52 & 1.5 & .0005 & 81.6 & 194 \\
7 & .80 & 0 & - & 32 & 165 \\
\hline
\end{tabular}

at each time step. In it we included the $I^{2} R$ losses in the resistors $R_{1}, R_{2}$ and the plasma shells, and the time integral of this quantity represents the total energy expended by the $O H$ system to drive the plasma up to a given state of current and temperature. In Figure 21 we display the results of temperature and current evolution calculations. In the lower figure, epoch marks have been shown on the resulting curves, corresponding to the times at whlch the expended resistive energy has reached $1.5,2.5$, and 5.0 Megajoules. It is clear from this data that the plasma evolution can be greatly enhanced by a short boost pulse. 
PLASMA CURRENT - $k$ A.

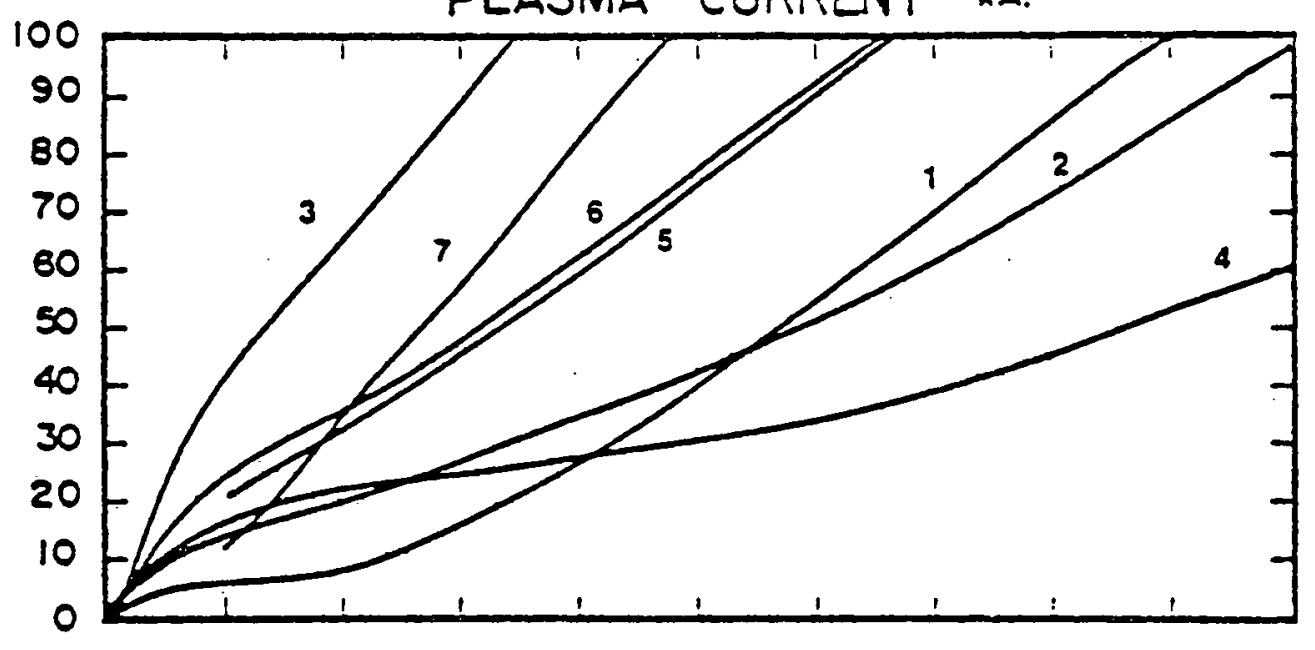

ELECTRON TEMPERATURE- $\mathrm{eV}$.

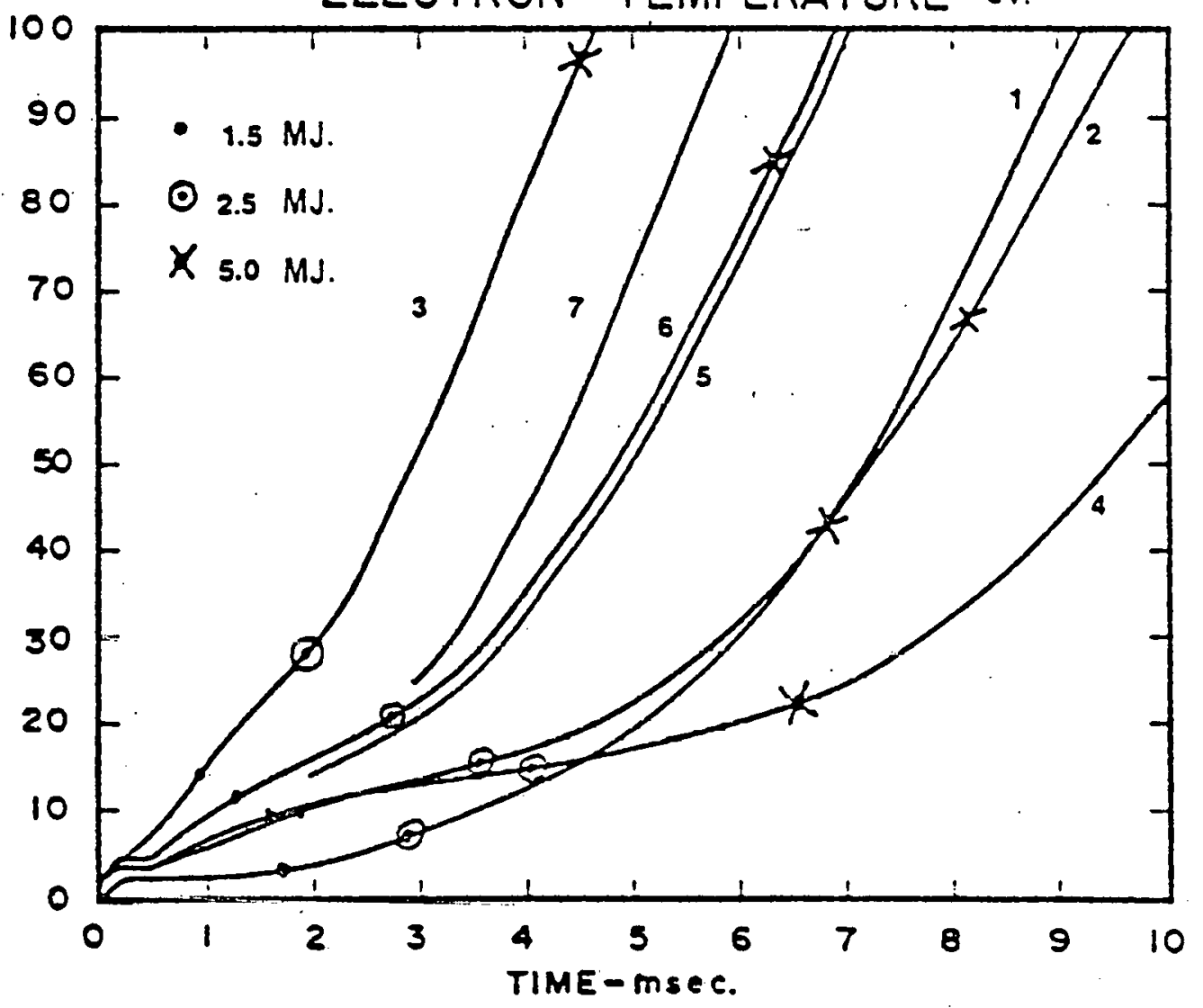

Figure 21. Plasma temperature and current evolution for boosted discharges. Epoch marks in. lower graph denote total resistive energy expenditure by $R 1, R 2$, and $R_{p}$. 
This is further illlustrated in Figure 22 , in which the electron temperature is plotted as a function of the $\mathrm{OH}$ energy expenditure for several cases. It would appear from this data that there is a potential saving of several MJ. of OH coil energy available by optimal selection of $R_{1}$ and $R_{2}$. However, as will be seen presently, this optimization is constrained by conditions relating to runaway electron production.

\section{B. Electron Runaway}

The criterion for runaway production was previously shown to be stated in the forms of equations (6-1) and $(6-2)$, where it can be seen that the criterion is a function of both electron and neutral densities. Both of these quantitites are rapidy changing during the first several hundred microseconds of the discharge. To quantify the potential for runaway production, we have defined a runaway parameter $\zeta$ by:

$$
\zeta=\frac{E}{E_{\text {cr. }}} \text {. }
$$

This quantity rapidly passes through a maximum in the first $50 \mathrm{\mu sec}$. Of the discharge as shown in Figure 23 for some typical cases, both boosted and unboosted. While it does appear that the runaway margin (i.e., $\zeta$ ) is lowest for the lowest values of peak plasma voltage, the total situation with regard to runaway 


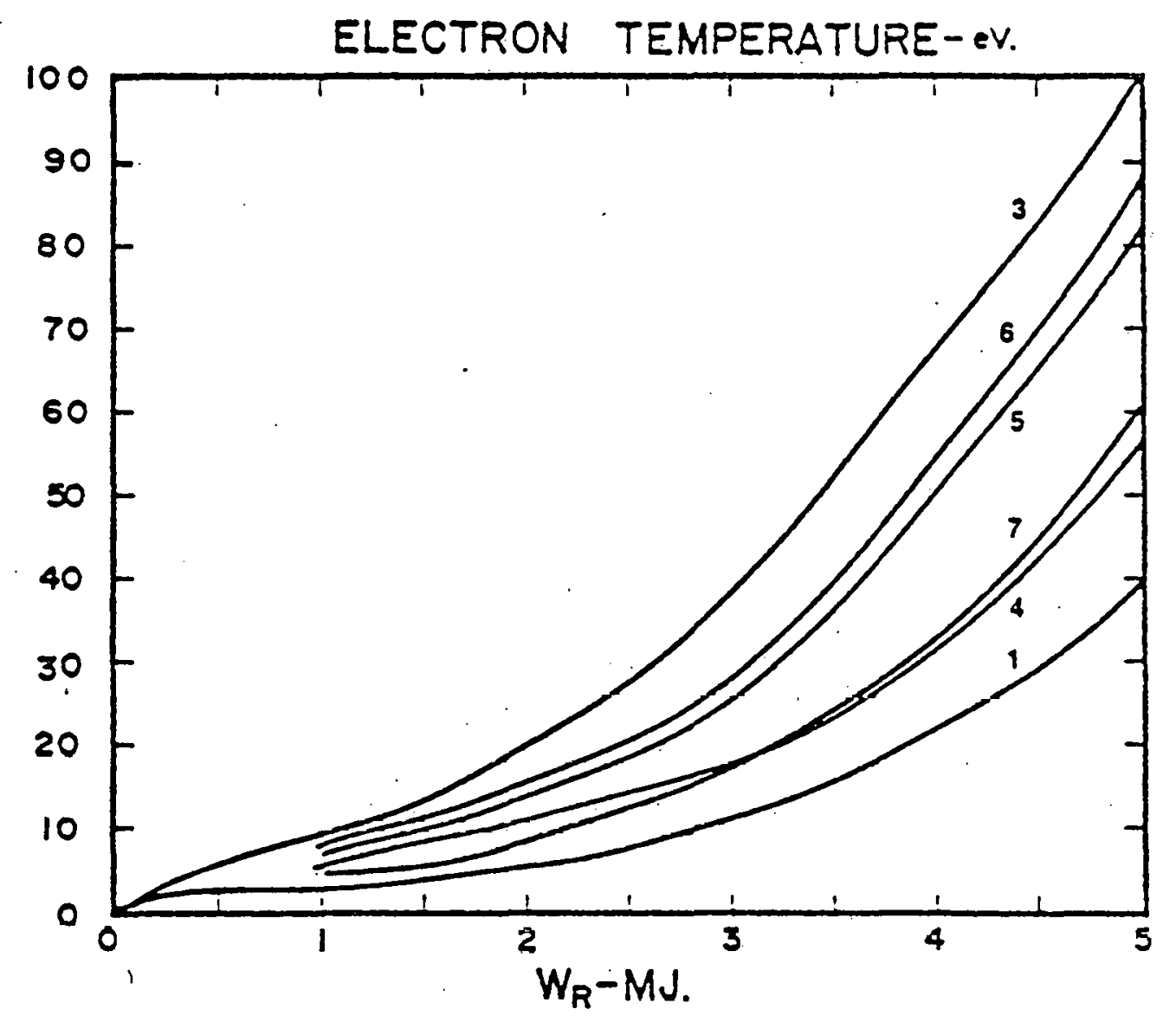

Figure 22. Electron temperature vs. resistive energy expenditure for various boosted and unboosted discharges. 


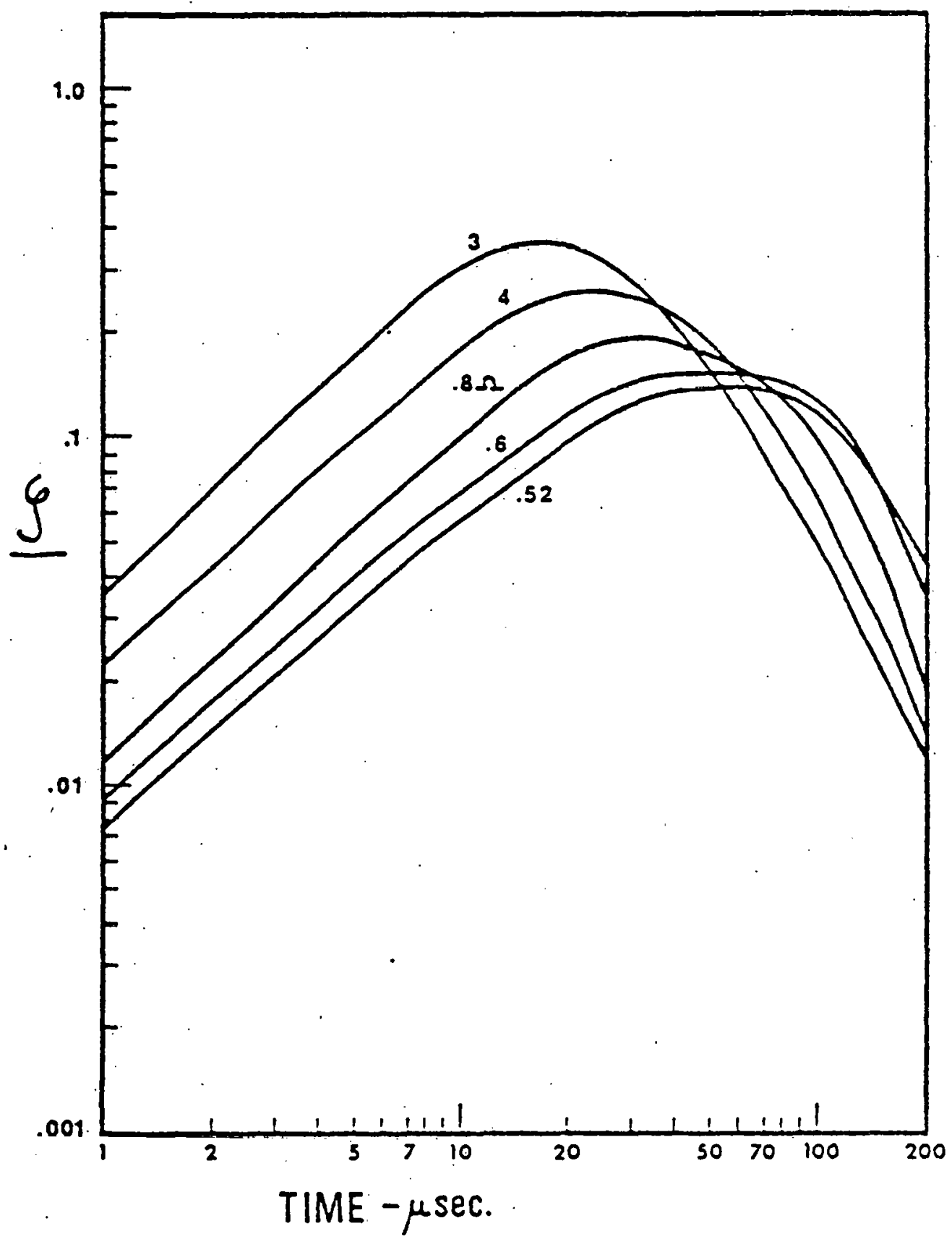

Figure 23. Runaway margin vs. time for several boosted and unboosted discharges. 
production is somewhat more complex than discussed so far.

Actually, runaway is not a clearly defined threshold process. At any value of $E$, a certain fraction of the electron population will runaway. More specifically, we can specify an arbitrary sphere in the electron velocity space which contains the main body of the electrons, and calculate (in principle!) the flux of electrons out of this region. The radius of this sphere corresponds to the "critical velocity." In the work of Gurevich (21), an approximate calculation was made of this flux although the results are somewhat difficult to calculate numerically. Now, we are concerned about the total production of runaways, which is going to involve an integral (tomporal) function of $\zeta$, which is quite rapidly varying during the first 100-200 microseconds. We have made an initial attempt to estimate the total runaway production, using the formulation of Gurevich. They indicate that the situation depicted by plots 3 and 7 in Figure 23 result in less total runaway fractions than for the remaining (unboosted) cases. This is due to the fact that the value of $\zeta$ is lower over a longer period of time (note the log scale in the figure) than it is higher. However, we add that these calculations as yet are quite primitive and require further refinement before the 
tentative conclusions stated here can be confidently accepted.

The preceeding discussion was based upon our results for a fixed initial ionization fraction, conservatively taken to be $.3 \%$. The time behavior of $\zeta$ is actually quite sensitive to this assumption, as shown in Figure 24, where the behavior of $\zeta$ is calculated for several initial pre-ionization levels. Also shown, for comparison, is the result of re-running case number 1 with its pre-ionization raised to $1 \%$. Recalling from Table 4 that case number 5 is the boosted version of case number 1 , it is notable that the boost decreases the energy required to reach a temperature of $100 \mathrm{eV}$. by a significant fraction. It also appears that if a pre-ionization level of $1 \%$ is practical, the runaway production may not be too severe and the boost will effectively decrease the required $O H$ energy store. We conclude the discussion by noting that runaway production is clearly related to the energetics of ohmic heating by placing a limit on the value of boost voltage which can be used to accelerate the ionization process. The details of the interaction between these two processes will require a more detailed. understanding of runaway kinetics, necessitating further analysis based upon the results contained in references 22 and 33. 


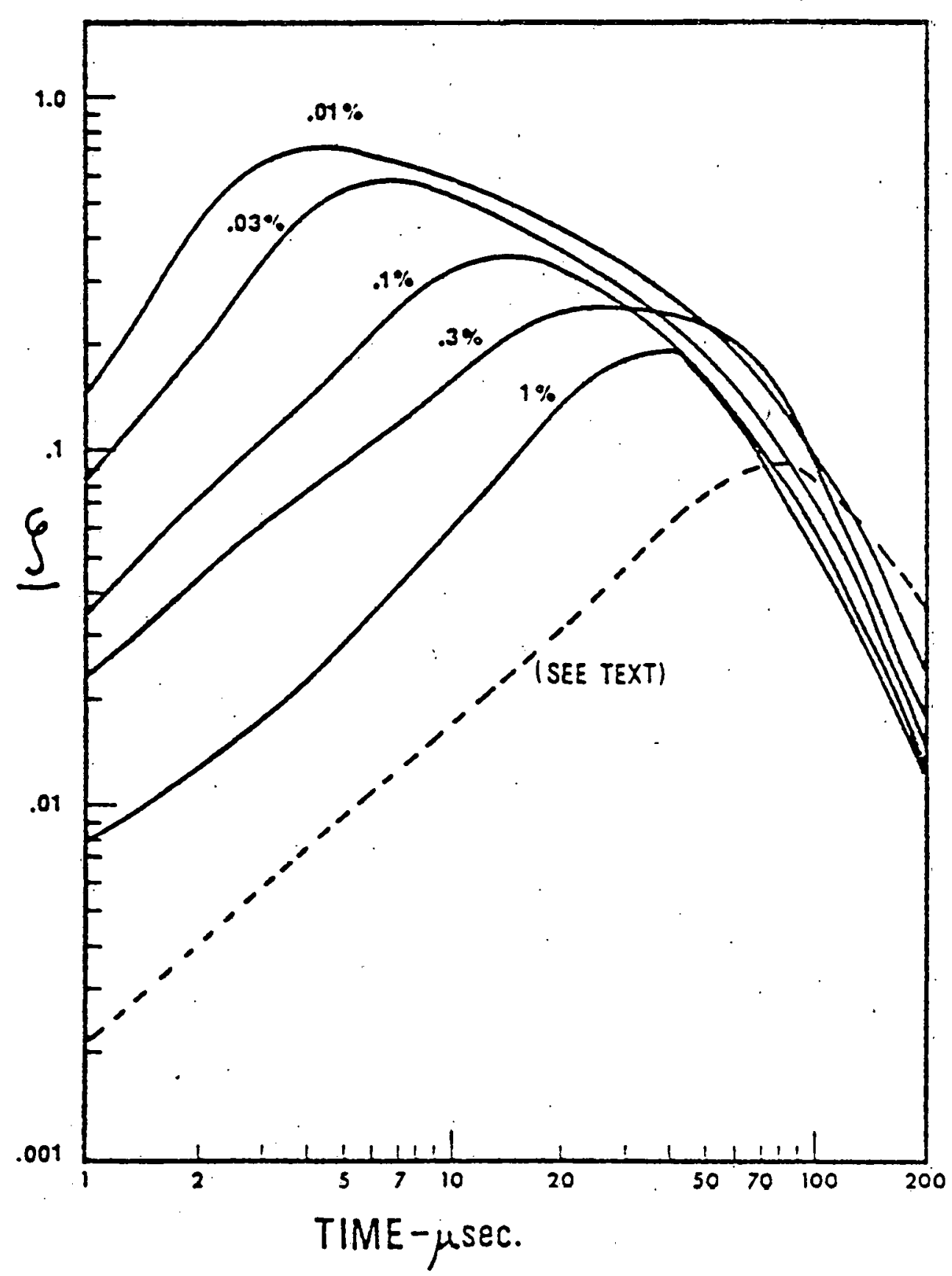

Figure 24. Runaway margin for different pre-ionization levels. Dashed curve is for parameters of case number 1 with pre-ionization increased to 18. 
77

4. Diffusion Simulation

The electrical formulation used in this study was developed primarily to deal with the problem of magnetic diffusion, which becomes more and more significant as the temperature rises above the $50 \mathrm{ev}$. point. As a result of this additional complication, zero-dimensional analysis of the plasma becomes more meaningless and ultimately fails totally to predict some of the important transient effects.

We begin the discussion by performing a simple analysis of the basic energy, diffusion processes in the plasma during and succeeding, for a short time, the initiation. The transport calculations of Hazeltine and Hinton (32) are utilized, and from the excellent review paper referenced, we obtain the expression for the cross-field heat flux:

$$
\begin{aligned}
\underline{Q}_{e c}= & -\frac{1}{m_{e} \Omega_{e}^{2} \tau_{e}}\left[.91 p_{e} \underline{\nabla}_{1} T_{e}-T_{e} \underline{\nabla}_{1} p_{e}\right] \\
\text { where } & p_{e}=M T_{e} \\
& \Omega_{e}=\frac{e B}{m c} \\
\tau_{e}= & \frac{3}{16 \sqrt{\pi}} \frac{m_{e}^{2} v_{T H}^{3}}{z^{2} e^{4} M_{i} \ln \Lambda}
\end{aligned}
$$

(11-1) 
78

from which the normal thermal conductivity coefficient is found to be:

$$
x_{\perp}=\frac{.91 m T_{e}}{\left[\sqrt{\frac{2}{T}} \cdot \frac{3}{8} \frac{T^{3 / 2} B^{2}}{c^{2} m_{e}^{1 / 2} e^{2} M \ln \Lambda}\right]} \quad\left(c s-e V_{1}\right)
$$

$(11-2)$

in which we have neglected the term due to the radial pressure gradient. The standard heat diffusion. equation is normally expressed in the form:

$$
\frac{\partial T_{e}}{\partial t}=a_{T}^{2} \nabla^{2} T_{e}
$$

when:

$$
a_{T}^{2}=\frac{\partial_{1}}{M_{e}}=\frac{2.8 \times 10^{-7} M_{e} \ln \Lambda}{B^{2} T^{3 / 2}}
$$

$(11-3)$

and the corresponding magnetic diffusion equation is:

$$
\frac{\partial B_{\theta}}{\partial t}=a_{m}^{2} \nabla^{2} B_{\theta}
$$

where from equation $(5-2) a_{M}$ is recalled to be:

$$
a_{m}^{2}=\frac{1}{\mu \sigma}=\frac{M}{\mu}=\frac{5.2 \times 10^{-3} \ln \Lambda}{4 \pi \times 10^{-9} T^{3 / 2}}=\frac{4.1 \times 10^{5} \ln \Lambda}{T^{3 / 2}}
$$

(11-4) 
A more physically meaningful interpretation of the diffusion constant a can be obtained by examining the Green's function for this equation, which for an n-dimensional distribution of the quantity $\psi$ is known to be of the form (24):

$$
\begin{gathered}
\psi(R, t)=\frac{4 \pi}{a^{2}}\left(\frac{1}{2 \sqrt{\pi\left(t-\tau_{0}\right)}}\right)^{m} e^{-\left(\frac{R^{2}}{4 a^{2}\left(t-t_{0}\right)^{2}}\right)} \\
R=\Lambda-\Lambda_{0} .
\end{gathered}
$$

From this it can be seen that injected sources will diffuse away from the source point $r_{0}$ and become uniformly distributed after a diffusion time which is approximately calculated as:

$$
\begin{aligned}
& 4 \tau \cong \frac{R^{2}}{a^{2}} \\
& \tau_{d} \cong \frac{R^{2}}{4 a^{2}}
\end{aligned}
$$

Comparing equations $(11-2)$ and $(11-3)$ we observe the following relationship between the diffusion times for magnetic field and electron heat energy (in reference 32 it is shown that the ion heat diffusion time is similar in magnitude) 
80

$$
\frac{a_{T}^{2}}{a_{m}^{2}}=\frac{2.83 \times 10^{-7} M_{e}}{4.13 \times 10^{5} B^{2}}=\frac{3.6 \times 10^{-13} M_{e}}{B^{2}}
$$

a result which is independent of the temperature but strongly dependent on the toroidal field $B_{T}$. For a typical case as in this study we find:

$$
\frac{a_{T}^{2}}{a_{m}^{2}} \simeq \frac{3.6 \times 10^{-13} \times 3 \times 10^{13}}{B^{2}} \sim \frac{10}{B^{2}}
$$

which for a toroidal field of $5.6 \mathrm{~T}$. (i.e., $56 \mathrm{kG}$.) implies

$$
\frac{\tau_{T}}{\tau_{M}} \sim 4 \times 10^{-9}
$$

Hence, for all practical purposes the radial heat flow vanishes above a temperature of a few lV. The purpose of this calculation is to show that a zero-dimensional model of the plasma as used here, in which it is assumed that all ohmic heat is instantaneously distribated throughout the plasma volume, is probably a poor model to use as the temperature approaches $100 \mathrm{eV}$. 
In particular, the slowing of the magnetic field penetration and the virtual absence of inward heat flux should lead to the formation of substantial skin current and an inverted temperature profile, which is quite unstable. Of course, we have assumed classical diffusion of the heat radially, which is known to a gross underestimate at higher temperatures where the neoclassical effects begin to dominate.

Using modules of code developed for the present study, we have made some introductory one-dimensional calculations of the early stages of ohmic heating, using the following simlifying assumptions:

(a) The plasma is modelled zero-dimensionally up to the state of full ionization.

(b) After full ionization is achieved, typically at a temperature of $3-4 \mathrm{ev}$. and a current level of $10 \mathrm{kA} .$, separate current and temperature calculations are performed in each plasma shell (i.e., a coarse radial grid is established).

(c) All cross-field heat transport is neglected.

(d) Impurity concentration is still handled zero-dimensionally.

(e) The radial density is assumed to remain constant. 
The main purpose of these calculations was to investigate the formation of skin current formation and profile inversion.

The simulations were run utilizing the same $O H$ system parameters as in the preceeding calculations, and a typical result is shown in Figure 25. The profiles are plotted as a function of the normalized minor radius at successive stages of evolution. The formation of the skin current and temperature profile inversion are quite pronounced as was expected. Further, a comparison of this data with the results of the same simulation performed previously in the zero-dimensional approximation, as shown in Figure 21, reveals that core of the plasma has heated at about the same rate but the outer layers have heated considerably faster. The total current evolution is about the same. Thus, it appears that we can successfully model the skin formation and current penetration in one-dimension. In our further studies, the one-dimensional formulation will be refined to include the appropriate transport and impurity effects. 

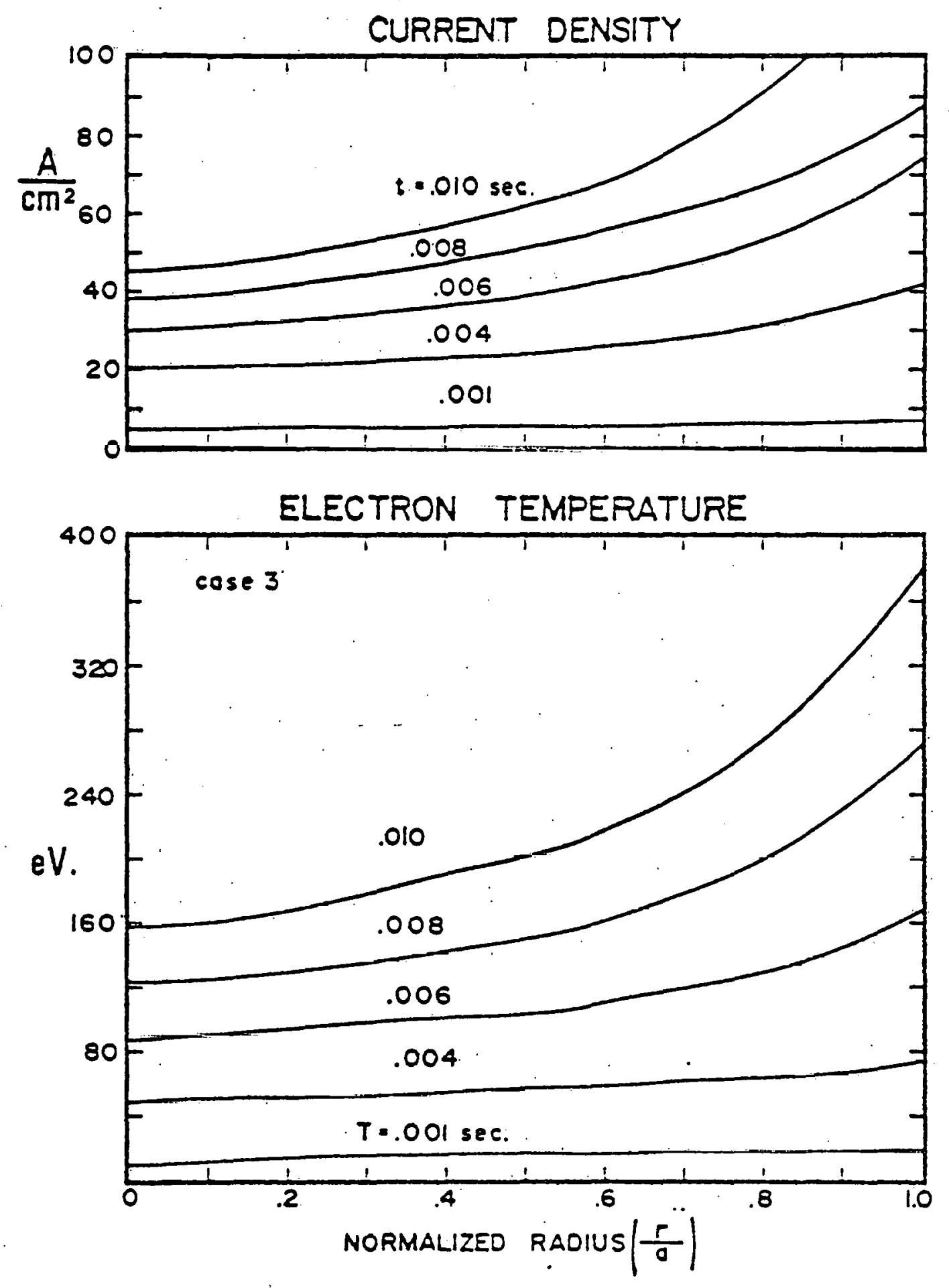

Figure 25. Temperature and current profiles in plasma at successive stages of heating, using parameters of case number 7 from Table 4. 


\section{APPENDIX A}

For calculation of induced plasma currents, it is generally useful to distinguish between the field induced by the plasma current itself and the field induced by external sources. The internal selfinductance is defined as the total flux inside of the plasma per unit of total plasma current, and the external self-inductance is defined as the total flux outside of the plasma per unit current. The external inductance is calculated by considering the entire plasma current to be concentrated along the toroidal axis, and calculating the resulting flux through the circle bounded by path 1 in the figure below:

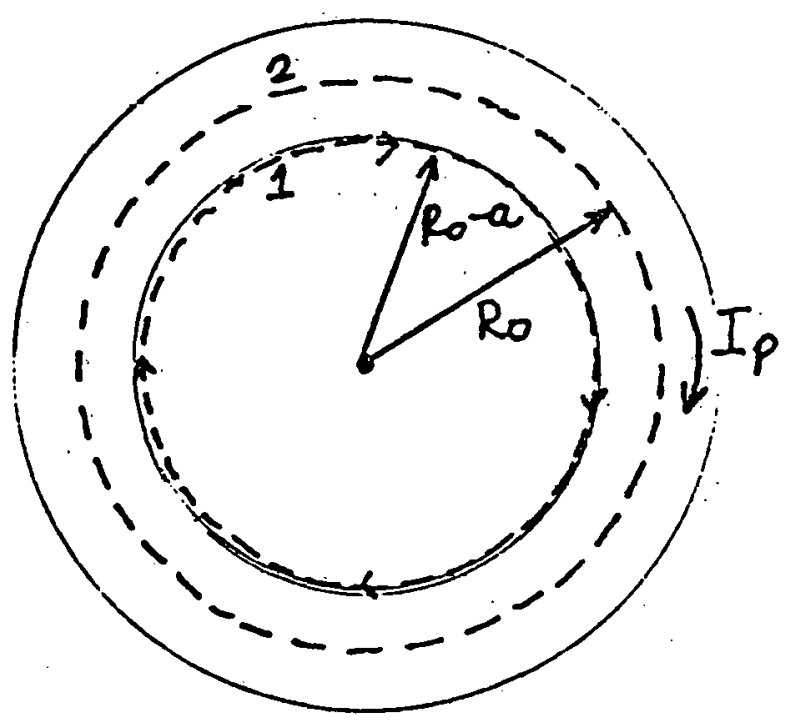


85

This flux calculation is identical to the one required to find the mutual inductance between thin conductors along path 1 and along the toroidal axis, for which the result is well known (30):

$$
\begin{aligned}
& M_{12}=\mu \sqrt{R_{0}\left(R_{0}-a\right)}\left[\left(\frac{2}{k}-k\right) K(k)-\frac{2}{k} E(k)\right] \\
& \text { where: } k^{2}=\frac{4\left(R_{0}-a\right) R_{0}}{\left[\left(R_{0}-a\right)+R_{0}\right]^{2}}
\end{aligned}
$$

and $K(k), E(k)$ denote the complete elliptic integrals. $k^{2}$ can be expanded to order $\varepsilon^{2}\left(\varepsilon=a / R_{0}\right)$ to yield:

$$
k^{2}=\frac{4(1-\epsilon)}{(2-E)^{2}} \simeq 1-\epsilon^{2}
$$

and the elliptic integrals can be approximated to similar order as (31):

$$
\begin{aligned}
& K(k) \simeq \ln \left(\frac{4}{\sqrt{1-k^{2}}}\right)=\ln \frac{4}{\epsilon}=\ln \left(\frac{4 R 0}{a}\right) \\
& E(k) \simeq 1
\end{aligned}
$$

$$
\Rightarrow m_{12}=L_{\text {eft }}=\mu R_{0}\left[\ln \left(\frac{8 R_{0}}{a}\right)-Z\right]
$$

A.1 
86

The internal inductance depends upon the assumed form of the current profile within the plasma, which we calculate for two commonly used cases as follows.

(i) Uniform current density: $j(r)=\frac{I_{p}}{\pi a^{2}}$

$$
\begin{aligned}
& H_{\theta}(\mu)=\frac{1}{2 \pi \nu} \cdot \frac{I_{p}}{\pi a^{2}} \cdot \pi \mu^{2}=\frac{I_{p}}{2 \pi a^{2}} \Omega \\
& \begin{aligned}
\Phi_{\text {int }} & =\mu \int_{0}^{a} H_{\theta}(\mu) \cdot 2 \pi R_{0} d \mu \\
& =\frac{\mu I_{p} R_{0}}{a^{2}} \int_{0}^{a} \sim d \mu=\frac{\mu I_{p} R_{0}}{2}
\end{aligned} \\
& L_{\text {int }}=\frac{\Phi_{\text {mint. }}}{I_{p}}=\frac{\mu R_{0}}{2} \quad A .2
\end{aligned}
$$


87

(ii) Pedestal current profile

$$
\begin{aligned}
& j(n)=\frac{I_{p}}{\pi a^{2}\left(1-\frac{c_{1}}{2}\right)}\left[1-C_{1}\left(\frac{\lambda}{a}\right)^{2}\right] \\
& H_{\theta}(\Omega)=\frac{I_{p}}{\pi a^{2}\left(1-\frac{1 l^{\prime}}{2}\right)} \cdot \frac{1}{2 \pi \Omega} \int_{0}^{N}\left[1-C_{1}\left(\frac{\lambda^{\prime}}{a}\right)^{2}\right] 2 \pi n^{\prime} d N^{\prime} \\
& =\frac{I_{p}}{2 \pi a^{2}\left(1-\frac{c_{1}}{2}\right)} \wedge\left[1-\frac{c_{1}}{2}\left(\frac{\lambda}{a}\right)^{2}\right] \\
& \Phi_{\text {int. }}=\frac{\mu I_{p}}{2 \pi a^{2}\left(1-\frac{c_{1}}{2}\right)} \int_{0}^{a} 2 \pi R_{0} \lambda\left[1-\frac{c_{1}}{2}\left(\frac{\mu}{a}\right)^{2}\right] d \sim \\
& =\frac{\mu I_{p} R_{0}}{a^{2}\left(1-\frac{c_{1}}{2}\right)}\left[\frac{a^{2}}{2}-\frac{c_{1}}{8} a^{2}\right] \\
& =\frac{\mu I_{p} R_{0}}{2\left(1-\frac{c_{1}}{2}\right)}\left(1-\frac{c_{1}}{4}\right) \\
& L_{\text {int }}=\frac{\Phi}{I_{p}}=\frac{\mu R_{0}}{2}\left(\frac{1-c_{1} / 4}{1-c_{1} / 2}\right)
\end{aligned}
$$




\section{LIST OF REFERENCES}

1. Dtlchs, D. F., Griem, H. R., Phys. Fluids 9 (1966) 1099 .

2. Hawryluk, R. J., Schmidt, J.A., Nuclear Fusion $16(1976) 775$.

3. Breton, C., De Michelis, C., Mattioli, M., Nuclear Fusion 16 (1976) 891.

4. Peng, M., Borowski, S. K., Kammash, T., Nuclear Fusion 18 (1978) 1489 .

5. TFTR Final Conceptual Design Report, Vol. I, TFTR-001, Princeton Plasma Physics Laboratory, p. 4-19I.

6. Kammash, T., Fusion Reactor Physics, Ann Arbor Science, Ann Arbor, 1977.

7. Lotz, W., Astroph. Journal Supplement Series No. 128,14 (1967) 207 .

8. Massey, H. S., Electronic and Ionic Impact Phenomenon, Vol. III, Clarendon Press, Oxford $(1967)$.

9. Duchs, D. F., Post, D. E., Rutherford, P. H., Nuclear Fusion 17 (1977) 565 .

10. Cox, D. P., Tucker, W. H., Astroph. Journal $155(1969) 27$.

11. Allen, J. W., Dupree, A. K., Astroph. Journal I55 (1969) 27 .

12. Griem, H. R., Plasma Spectroscopy, McGraw-Hill, New. York $(1965)$.

13. Tucker, W. H., Gould, R. J., Astroph. Journal 144 (1966) 244 .

14. Wiese, W. I., Smith, M. W., Glennon, B. M., Atomic Transition probabilities, Vol. I, NSRDS (1966). 
15. Holstein, T., Phys. Rev. 70 (1946) 367.

16. Hawryluk, R. J. (unpublished, 1978) communicated to the author by $G$. Gibson of Westinghouse Fusion Power Systems.

17. Spitzer, L., Astroph. Journal 107 (1948) 6 .

18. Bernstein, Chen, Heald, Krantz, Phys. Fluids 1 (1958) 430 .

19. Dreicer, H., Phys. Rev. 115 (1959) 238.

20. Dreicer, H., Phys. Rev. 117 (1960) 329.

21. Gurevich, A. V., Soviet Physics JETP, 12 (1961) 904.

22. Spong, D., ORNL TM-5147 (March 1976).

23. Rosenbluth, M. N., Iiu, C. S., Phys. Fluids 19 (1976) 815 .

24. Morse, P. M., Feshbach, H., Methods of Mathematical Physics, McGraw-Hill, New York (1953):

25. Hawryluk, R. J., Schmidt; J.A. (unpublished memo from $\mathrm{P}^{3} \mathrm{I}_{\text {.) }}$

26. von Valtier, E. (unpublished).

27. see ref. 5, p. 4-40.

28. Malmberg, J. H., Rosenbluth, M. N., Rev. Scientific Instruments, 36 (1965) 1886 .

29. see ref. 16 .

30. Ramo, S., Whinnery, J. R., van Duzer, T., Fields and Waves in Communications Electronics, wiley (1967).

31. Hart, J. F. et al, Computer Approximations, Wiley, New York (1968).

32. Hazeltine, R. D., Hinton, F. I., Rev. Modern Phys. 48 (1976) 239 .

33. Mok, Y., Ph.U. thesis, U. of Maryland, 1979. 